\title{
RIPK3 collaborates with GSDMD to drive tissue injury in lethal polymicrobial sepsis
}

\author{
Hui Chen ${ }^{1} \cdot$ Yinshuang $\mathrm{Li}^{1} \cdot$ Jianfeng $\mathrm{Wu}^{2} \cdot$ Guoping $\mathrm{Li}^{3} \cdot \mathrm{Xuan}^{\mathrm{Tao}}{ }^{3} \cdot \mathrm{Kunmei}^{\mathrm{Lai}}{ }^{1} \cdot$ Ying Yuan $^{1} \cdot \mathrm{Xiaohong}_{\mathrm{Zhang}}{ }^{1}$. \\ Zhenhuan $\mathrm{Zou}^{1} \cdot$ Yanfang $\mathrm{Xu}^{1}$
}

Received: 12 November 2019 / Revised: 21 February 2020 / Accepted: 21 February 2020 / Published online: 9 March 2020

(c) The Author(s), under exclusive licence to ADMC Associazione Differenziamento e Morte Cellulare 2020

\begin{abstract}
Sepsis is a systemic inflammatory disease causing life-threatening multi-organ dysfunction. Accumulating evidences suggest that two forms of programmed necrosis, necroptosis and pyroptosis triggered by the pathogen component lipopolysaccharide (LPS) and inflammatory cytokines, play important roles in the development of bacterial sepsis-induced shock and tissue injury. Sepsis-induced shock and tissue injury required receptor-interacting protein kinase-3 (RIPK3) and mixed lineage kinase domain-like protein (MLKL) phosphorylation, caspase11 activation and gasdermin D (GSDMD) cleavage. However, the synergistic effect of necroptosis and pyroptosis in the pathological progress of sepsis remains elusive. In this study, we found that blockage of both necroptosis and pyroptosis (double deletion of Ripk3/Gsdmd or Mlkl/Gsdmd) resulted in accumulative protection against septic shock, systemic blood clotting and multi-organ injury in mice. Bone marrow transplantation confirmed that necroptosis and pyroptosis in both myeloid and nonmyeloid cells are indispensable in the progression of sepsis-induced multi-organ injury. Both RIPK3 and GSDMD signaling collaborated to amplify necroinflammation and tissue factor release in macrophages and endothelial cells, which led to tissue injury. Furthermore, cell death induced by inflammatory cytokines and high-mobility group box 1 could be prevented by double ablation of Ripk3/ Gsdmd or Mlkl/Gsdmd, suggesting that a positive feedback loop interconnecting RIPK3/MLKL and GSDMD machinery and inflammation facilitated sepsis progression. Collectively, our findings demonstrated that RIPK3-mediated necroptosis and GSDMD-mediated pyroptosis collaborated to amply inflammatory signaling and enhance tissue injury in the process of sepsis, which may shed new light on two potential targets of combined therapeutic interventions for this highly lethal disorder.
\end{abstract}

These authors contributed equally: Hui Chen, Yinshuang Li

Edited by T. Mak

Supplementary information The online version of this article (https:// doi.org/10.1038/s41418-020-0524-1) contains supplementary material, which is available to authorized users.

Yanfang Xu

xuyanfang99@hotmail.com

1 Department of Nephrology, First Affiliated Hospital, Fujian Medical University, Fuzhou 350005, China

2 School of Life Sciences, Xiamen University, Xiamen 361005, China

3 Department of Pathology, First Affiliated Hospital, Fujian Medical University, Fuzhou 350005, China

\section{Introduction}

Sepsis, defined as life-threatening organ dysfunction resulting from systemically altered host response to infection, is still a major challenge for healthcare systems [1]. Despite the situation has been improved during these years, there is still no approved treatment specifically for sepsis. Unlike nonseptic critical illness, sepsis is infection-induced systemic inflammatory response syndrome (SIRS) due to excessive activation of immune cells and overproduction of inflammatory cytokines, which ultimately results in the dysfunction or damage of multiple organs [2-4]. Its underlying mechanisms may involve complex systemic inflammatory network effects [5], genetic polymorphisms [6], coagulopathy [7], immune dysfunction [8], tissue damage, and abnormal responses of the host to different infectious pathogenic microorganisms and their toxins [9].

Recently, the roles of programmed necrosis (necroptosis and pyroptosis) in host response to infectious pathogens 
have gained considerable attention. Necroptosis and pyroptosis represent two distinct pathways of genetically encoded necrotic cell death. In one hand, these cell death programs protect the host against microbial pathogens. In the other hand, such lytic cell death modes that are distinct from apoptosis may result in the release of various intracellular components including damage-associated molecular patterns (DAMPs) and inflammatory cytokines such as interleukin-1 $\beta$ (IL-1 $\beta$ ) to self-amplify or even trigger the inflammatory process [10]. In fact, genetic evidences indicated that these cell death pathways, once activated, can induce potent inflammatory responses in vivo and thereby contribute to pathogenesis of various inflammatory diseases [11-14]. It is noteworthy that although these two modes of lytic cell death appear quite similar, necroptosis and pyroptosis may function in different contexts. For instances, necroptosis can serve as an efficient backup cell death mechanism upon infections, in which pathogens apply caspase-inhibitory proteins or upregulation of receptorinteracting protein kinase-3 (RIPK3) to block apoptosis $[15,16]$. RIPK3-mediated necroptosis could be triggered by biological stimuli, including TNF and interferons (IFNs). On the other hand, pyroptosis occurring upon activation of proinflammatory caspases and their subsequent cleavage of gasdermin D (GSDMD), is a primary cellular response to various cellular insults, including pathogen-associated molecular patterns, DAMPs, altered levels of host metabolites and environmental irritants [10].

RIPK3 is an essential mediator for necroptosis. Upon activation, RIPK3 interacts with RIPK1 through RIP homotypic interaction motif, then phosphorylates and activates the executioner "mixed lineage kinase domain-like" (MLKL), which forms pore in cytoplasmic membrane [17]. Early studies have shown that genetic ablation of Ripk3 not only conferred protection against $\mathrm{TNF} \alpha$-induced lethal SIRS but also to certain extent, protected mice from the cecal ligation and puncture (CLP)-induced sepsis, demonstrating a crucial role of RIPK3 in sepsis [18, 19]. RIPK1 kinase-inactive Ripk1 $1^{D 138 N / D 138 N}$ mice could be protected from TNF-induced SIRS through prevention of endothelial cell (EC) necroptosis, confirming that necroptosis plays an important role in the sepsis progression [20]. Besides, both Caspase-11 and its propyroptotic substrate GSDMD, the terminal effector of pyroptosis, have been implicated in in endotoxemia. Deficiency of Caspase-11 or Gsdmd protected mice from lipopolysaccharide (LPS)-induced lethal septic shock although CLP model of sepsis was not examined in the study [21]. However, whether RIPK3/ MLKL-induced necroptosis could collaborate with GSDMD-mediated pyroptosis to execute organ dysfunction during sepsis remains an open question. In this study, we report that both necroptosis and pyroptosis are engaged in CLP-induced sepsis and multi-organ dysfunction. Genetic ablation of Ripk3/Gsdmd or Mlkl/Gsdmd provides a synergistic protection against septic shock and multi-organ damage.

\section{Materials and methods}

\section{CLP-induced sepsis model}

Ripk3 $^{-1-}, \mathrm{Mlkl}^{-1-}$, and $\mathrm{Gs} d \mathrm{md}^{-/-}$mice of C57BL/6 genetic background were gifts from Dr. Jiahuai Han's Laboratory, School of Life Sciences, Xiamen University, China. Ripk3/

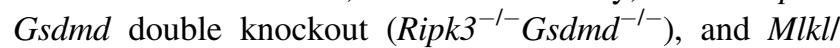
Gsdmd double knockout ( $M l k l^{-1-} G s d m d^{-1-}$ ) mice were generated by intercrossing of these strain. Genotypes were determined by tail-snip PCR amplification. Mice were housed in a specific pathogen-free facility with 12-h light/ dark cycles. All the experiments were conducted according to the Chinese Guidelines on the Care and Use of Laboratory Animals, with approval from Laboratory Animal Management and Ethics Committee of Fujian Medical University. The CLP procedure was performed in male mice (8- to 12 -week-old, 22-26 g body weight) by a skillful surgeon according to the guidelines [22]. Briefly, after mice were anesthetized with ketamine $(80-100 \mathrm{mg} / \mathrm{kg} / \mathrm{i} . \mathrm{p}$., Cayman Chemical) and xylazine $(10 \mathrm{mg} / \mathrm{kg} / \mathrm{i} . p .$, Selleck Chemicals), a 10-mm midline abdominal incision was performed and the cecum exposed. The cecum was ligated with $4-0$ silk at $75 \%$ of the distance between distal pole and the base (ileocecal valve), then perforated by a single through-and-through puncture with a 20 -gauge needle. The cecum was slightly compressed to extrude a small amount (droplet) of feces. The abdomen was closed in two layers with 5-0 suture for the peritoneum and abdominal musculature. Following surgery, the mice were injected with prewarmed physiological saline solution $\left(37^{\circ} \mathrm{C} ; 1 \mathrm{ml}\right.$ per $20 \mathrm{~g}$ body weight) subcutaneously (s.c.) and kept warm at $37^{\circ} \mathrm{C}$ under the lamp until they recovered from anesthesia. At the end of the surgical procedures, mice were put back in cages in a temperature-controlled room $\left(25^{\circ} \mathrm{C}\right)$ where free access to water and food was available. Mice were monitored closely. Pre- and postoperative analgesia (Ibuprofen, $200 \mu \mathrm{g} / \mathrm{ml}$ in drinking water) were given to the mice from $24 \mathrm{~h}$ before and until $48 \mathrm{~h}$ after surgery. After surgery, mice were randomly and blindingly sacrificed at different time points to assess multi-organ dysfunction. Blood and various organs including liver, lung, kidney, and intestine were collected and analyzed at the indicated time points.

For chimera studies, recipient mice were irradiated by lethal X-ray irradiation $(7.5 \mathrm{~Gy})$, and then $2 \times 10^{6}$ donor bone marrow $(\mathrm{BM})$ cells isolated from donor mice were injected into recipients via the tail vein. 


\section{Reagents and antibodies}

Anti-RIPK3, anti-p-RIPK3, anti-MLKL, anti-pMLKL, and anti-GSDMD antibodies for western blot, and anti-p-RIPK3 for immunohistochemical staining and mouse recombinant $\mathrm{TNF} \alpha(\mathrm{mTNF} \alpha)$ were a kind gift from Dr. Jiahuai Han. Anti-pMLKL (phospho S345) antibody (ab196436, abcam), anti-F4/80 antibody [CI: A3-1] (ab6640, abcam), anti-Ly6G antibody [RB6-8C5] (ab25377, abcam) were used for immunohistochemical staining. Anti-mouse IL-1 $\beta$ antibody (5129-100, BioVision), anti-caspase-11 antibody (17D9, Vovus Bio), anti-ICAM (ab25375, Abcam), anti-GAPDH (3781, ProSci), anti-tubulin (T9026, Sigma-Aldrich) were used for western blotting. Anti-Fibrinogen antibody (ab27913, Abcam) was used for western blotting and immunohistochemistry. Mouse TNF $\alpha$ (MTA00B), IL-6 (M6000B), IL-1 $\beta$ (MLB00C), and IFN $\beta$ (42400-1) ELISA Kit provided by R\&D Systems, high-mobility group box 1 (HMGB1) ELISA (ST51011) abtained from TECAN were used for detection their secretion levels. AssaySense TF Chromogenic Activity Assay kit (CT1002b, Assaypro) was used for tissue factor (TF) activity detection. V500 (65-0867-14), Fixable Viability Dye eFluorTM 520, was purchased from eBioscienceTM. ApopTag fluorescein in situ apoptosis detection kit (S7110) was purchased from EMD Millipore.

\section{Histological analysis}

Lung, intestine, liver, and kidney were fixed in $4 \%$ normal formalin. Paraffin sections $(4 \mu \mathrm{m})$ were stained with $\mathrm{HE}$, PAS or TUNEL reagent (ApopTag Fluorescein In Situ Apoptosis Detection Kit) according to the manufacturer's instructions. Routine protocols were performed for immunohistochemical staining. The number of F4/80-positive or Ly6G-positive cells were determined with Image J software.

For morphologic quantifications, at least ten random visual fields under microscopy $(\times 100)$ of per lung, small intestine, liver, or kidney section were analyzed. Lung injury scores were assessed based on the following parameters: alveolar capillary congestion, hemorrhage, inflammatory cell infiltration, alveolar wall thickness and hyaline membrane formation. The percentage of lung injury was counted on a scale of $0-10$ : 0 , not present (normal); $1-4$, 10-40\% (mild); 5-6, 50-60\% (moderate); 7-8, 70-80\% (severe); 9-10, 90-100\% (very severe). The damage of small intestine was scored by counting the percentage of intestinal epithelium atrophy marked by sloughed and shed cells with condensed nuclei as follows: 0 , normal; 1 , $1-10 \%$; 2, 11-25\%; 3, 26-50\%; 4, 51-75\%; 5, $\geq 75 \%$. To assess the degree of intravascular coagulation, we calculated the percentage of blood vessels containing clotting in the liver blood vessels. Morphological damage of PAS-stained kidney sections was evaluated by following parameters: brush border loss, tubular dilation, tubular necrosis, and cast formation. The percentage of these parameters were counted on a scale of 0-10: 0 , not present (normal); $1-4,10-40 \%$ (mild); 5-6, 50-60\% (moderate); 7-8, 70-80\% (severe); $9-10,90-100 \%$ (very severe).

\section{Flow cytometry}

Single-cell suspensions from blood, lung, kidney and liver were stained with cell-surface antibodies for F4/80 (254801-82, eBioscience ${ }^{\mathrm{TM}}$ ), and Ly-6G/Ly-6C (RB6-8C5, 115931-82, eBioscience ${ }^{\mathrm{TM}}$ ) after blocking of $\mathrm{Fc}$-mediated reactions by using CD16/CD32 Monoclonal Antibody 93 (14-0161-85, eBioscience ${ }^{\mathrm{TM}}$ ). V500 (eBioscience ${ }^{\mathrm{TM}}$ Fixable Viability Dye eFluor $\left.{ }^{\mathrm{TM}} 520,65-0867-14\right)$ was used to distinguish between live and dead cells. To obtain single-cell suspension, tissues were digested with a cocktail of $1 \mathrm{mg} / \mathrm{ml}$ collagenase D (Roche, 11088858001) and $200 \mu \mathrm{g} / \mathrm{ml}$ DNase I (Roche, 11284932001) in PBS at $37{ }^{\circ} \mathrm{C}$ for $30 \mathrm{~min}$. All samples were run on a Navios (Beckman) flow cytometer and analyzed using FlowJo software.

\section{Hematologic analysis}

At indicated times after CLP, peritoneal blood cells were harvested in an EDTA-coated tube. Differential cell counts were determined using an automated hematology analyzer (Siemens, ADVIA2120i).

\section{Neutrophil cell death}

For peripheral neutrophil cell death analysis, after fresh blood cells were harvested, Red Blood Cell Lysing Buffer (Beyotime Biotechnology) was used to remove red cells. After being washed with isolation buffer, the cells were resuspended in discontinuous Percoll/HBSS gradients (52, 64 , and $72 \%$ ) and neutrophil population was obtained at the $64-72 \%$ interface. The purified neutrophils were resuspended in RPMI 1640 medium containing 10\% heatinactivated FBS. After indicated time periods, cells were harvested, washed with ice-cold PBS containing 1\% BSA then stained with V500 followed by FACS analysis using Navios (Beckman) flow cytometer. V500 positive cells indicated the death of neutrophils.

\section{RNA analysis}

Total RNA was obtained from the lung by RNA-iso reagent (TakaRa). Reverse Transcription Kit (BGI, Shenzheng, China) was used to reverse-transcribe total RNA to cDNA. The levels of Intercellular adhesion molecule-1 (ICAM-1) and $\beta$-actin were determined by SYBR Green I Real time 
quantitative PCR in a CFX96 real-time RT-PCR detection system (Bio-Rad). PCR amplification was carried out for 41 cycles. The primer sequences described as before [23] were obtained from Sangon Biotech (Shanghai, China).

\section{Western blot analysis}

Primary cultured cells were harvested and then lysed with $1.2 \times$ SDS buffer immediately. Approximate $30-50 \mathrm{mg}$ tissues were placed in 300-500 $\mu$ l RIPA lysis buffer containing protease inhibitors cocktail and then homogenized. Bradford protein assay was used for detecting protein concentrations and then SDS was added. The lysates were subjected to electrophoresis and separated by SDS-PAGE, then transferred to polyvinylidene fluoride membranes (EMD Millipore). The blots were visualized with ECL method.

\section{Cell culture}

Bone marrow derived macrophages (BMDMs) were obtained from the tibia and femur and then cultured in RPMI 1640 containing 30\% L929-conditional medium for differentiating BM progenitors. After 6-7 days, BMDMs were divided and plated in 12-well tissue plate for $24 \mathrm{~h}$. For necroptosis assessment, (i) BMDMs were pretreated with $1 \mu \mathrm{g} / \mathrm{ml}$ LPS for $3 \mathrm{~h}$ and then preincubated with $10 \mu \mathrm{M}$ QVD-OPh (without inhibiting caspase-1 activity, R\&D Systems) for $30 \mathrm{~min}$ before stimulation with $10 \mu \mathrm{M}$ Smacmimetic [antagonizes inhibitor of apoptosis proteins (IAPs)] (LCL-161, TargetMol) for $5 \mathrm{~h}$; (ii) BMDMs were treated with $30 \mathrm{ng} / \mathrm{ml} \mathrm{TNF} \alpha, 10 \mu \mathrm{g} / \mathrm{ml} \mathrm{CHX}$, and $10 \mu \mathrm{M}$ Smacmimetic $(\mathrm{TNF} \alpha+\mathrm{C}+\mathrm{S})$ for $24 \mathrm{~h}$. For pyroptosis assessment, (i) BMDMs were pretreated with LPS $(1 \mu \mathrm{g} / \mathrm{ml})$ for $3 \mathrm{~h}$ and then treated with $15 \mu \mathrm{M}$ Nigericin for $1 \mathrm{~h}$; (ii) treated $2 \mu \mathrm{g} / \mathrm{ml}$ LPS and $500 \mathrm{ng} / \mathrm{ml}$ HMGB1 (Fully reduced HMGB1, TECAN REHM114) for $16 \mathrm{~h}$; (iii) primed with $1 \mu \mathrm{g} / \mathrm{ml}$ Poly(I:C) (HMW, Invitrogen) and then transfected with LPS $2 \mu \mathrm{g} / \mathrm{ml}$ LPS (E.coli 0111:84 LPS-EB Ultrapure, Invitrogen). FuGENE HD (E2311, Progema) was used for LPS transfection.

Mouse lung microvascular ECs were obtained as previously described. In brief, blood-free mouse lungs were placed in $0.25 \%$ Tyrisin-EDTA at $37^{\circ} \mathrm{C}$ for $5 \mathrm{~min}$, then minced and digested with $1.5 \mathrm{mg} / \mathrm{ml}$ Collagenase D (Cata$\log$ No. 11088866001, Sigma-Aldrich) and $1 \mathrm{mg} / \mathrm{ml} \mathrm{Col-}$ lagenase/Dispase (Catalog No. 10269638001, SigmaAldrich) at $37^{\circ} \mathrm{C}$ for $30 \mathrm{~min}$ and centrifuged. ECs were magnetically sorted after cultured with PECAM-1-coated Dynabeads. Isolated ECs were cultured in ECM (Catalog No. 1001, Sciencell) containing endothelial growth supplement on the plate precoated with collagen (Catalog No.
9007-34-5, Sigma-Aldrich). Cells were characterized by FACS analysis using EC surface markers including antiCD31, anti-VE-cadherin, and anti-VEGFR2 antibodies.

For EC necroptosis assessment, ECs were treated with $30 \mathrm{ng} / \mathrm{ml} \mathrm{TNF} \alpha, 10 \mu \mathrm{g} / \mathrm{ml} \mathrm{CHX}$ and $10 \mu \mathrm{M}$ Smac-mimetic $(\mathrm{TNF} \alpha+\mathrm{C}+\mathrm{S})$ for $24 \mathrm{~h}$. For EC pyroptosis assessment, (i) ECs were treated $2 \mu \mathrm{g} / \mathrm{ml}$ LPS and $500 \mathrm{ng} / \mathrm{ml} \mathrm{HMGB} 1$ for $20 \mathrm{~h}$; (ii) primed with $500 \mathrm{ng} / \mathrm{ml}$ LPS for $3 \mathrm{~h}$ and then transfected with $2 \mu \mathrm{g} / \mathrm{ml}$ LPS. FuGENE HD was used for LPS transfection. To confirmed ECs undergoing pyroptosis, GSDMD-N-HBD-HA-reconstituted cells were added with $1 \mu \mathrm{M}$ 4-hydroxytamoxifen (4-OHT). HA tag was fused to the C-terminus of GSDMD-N-HBD for immunostaining analysis. For cell death determination, cells were stained with propidium iodide (PI) to indicate cell death and Hoechst to visualize nuclei. PI negative cells indicated cell survival.

To assess EC permeability, ECs were treated TNF $\alpha$ plus $\mathrm{CHX}$ and Smac-mimetic ( $\mathrm{TNF} \alpha+\mathrm{C}+\mathrm{S})$, or $\mathrm{TNF} \alpha$ plus IFN $\beta$, or primed with $1 \mu \mathrm{g} / \mathrm{ml}$ LPS for $5 \mathrm{~h}$ firstly and then added with medium from LPS-transfected BMDMs. SYTOX Green (Catalog No. S7020, Sigma-Aldrich) nucleic acid stain was added to the cells as the indicated treatment, and then measured using an IncuCyte instrument (Essen Bioscience).

\section{Lentivirus preparation and infection}

For generation of recombinant lentivirus, $293 \mathrm{~T}$ cells were cotransfected with expressing lentivirus-mix packaging plasmids (PMDL/REV/VSVG) and a pBOB construct encoding a given cDNA by calcium phosphate precipitation method. pBOBI-Flag-RIPK3, pBOBI-GSDMD, and pBOBI-GSDMD-N-HBD-HA were provided by Dr. Jiahuai Han. The virus-containing medium was harvested $40-44 \mathrm{~h}$ later and then added to ECs with $10 \mu \mathrm{g} / \mathrm{ml}$ polybrene for increasing infectious efficiency. Infectious medium was changed $14-16 \mathrm{~h}$ later. After $40-48 \mathrm{~h}$, the infected cells were used for further experiments.

\section{Immunofluorescence imaging}

ECs were grown on coverslips precoated with poly-Llysine (Sigma-Aldrich). After treatment, cells were stained with PI firstly to show the dead cells. After washed with PBS, cells were fixed in $4 \%$ paraformaldehyde for $15 \mathrm{~min}$ then permeabilized by $0.25 \%$ Triton X-100/PBS. After blocking with $10 \%$ normal goat serum, cells were incubated with anti-HA antibody, followed by secondary antibodies. Afterwards, cells were stained with DAPI to visualize nuclei. Imaging was carried out using Zeiss LSM 780. 


\section{Vascular permeability}

For lung vascular permeability assessment, $1 \%$ Evans blue (E2129, Sigma-Aldrich) solution was injected i.v. at $40 \mathrm{mg} /$ $\mathrm{kg} 22 \mathrm{~h}$ after CLP. After $1 \mathrm{~h}$ Evans blue treatment, mice were sacrificed, then perfused with ice-cold PBS. Lungs were homogenized in formamide after dried overnight. The amount of extracted Evans blue dye was evaluated by a spectrophotometer with a Beckman Coulter DTX880 plate reader.

For determination of intestinal permeability, mice were orally gavaged with $100 \mu \mathrm{l}$ of $150 \mathrm{mg} / \mathrm{kg}$ FITC-dextran (average mol wt 4000) beads (46944, Sigma-Aldrich) $12 \mathrm{~h}$ after CLP. Six hours after FITC-dextran administration, blood was drawn via cardiac puncture and harvested in an EDTA-coated tube. The relative amount of FITC-dextran in plasma was measured by fluorometry on a Beckman Coulter DTX880 plate reader.

\section{Statistical analysis}

All experiments were independently performed in triplicate as a minimum. All data are shown as means \pm SEM. Statistical analysis was performed with Prism software (GraphPad Software, Inc.). Unpaired Student's $t$ tests or two-way analysis of variance test with Bonferroni post-test was used to compare the means of two groups. $P<0.05$ was considered to indicate statistical significance.

\section{Results}

\section{Combined deletion of Ripk3 and Gsdmd confers cumulative protection against CLP-induced sepsis}

To determine whether necroptosis and pyroptosis have cumulative effects in sepsis, the Ripk3/Gsdmd double knock-

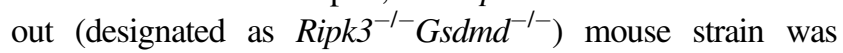
generated by intercrossing Ripk $3^{-/-}[24,25]$ and $G s d m d^{-/-}$ [26] mouse strains, both of which were maintained on a C57BL/6 genetic background. The Ripk $3^{-1-} \mathrm{Gsdmd}^{-1-}$ mice are viable and fertile with no overt pathology under stress-free conditions (data not shown). We performed a severe CLP on age- and sex-matched mice with a 20 -gauge needle. Wild-type (WT) mice exhibited a sharp drop in body temperature and almost did not recover from the initial hypothermia. Ripk $3^{-1-}$ or $G s d m d^{-1-}$ mice showed a gradual drop in body temperature, but started to recover at $36 \mathrm{~h}$ after CLP. Interestingly, the body temperature of $\mathrm{Ripk}^{-1-} \mathrm{Gsdm}^{-1-}$ mice was significantly higher than $R_{i p k} 3^{--}$or Gsdmd ${ }^{-1-}$ single knockout mice at all the time points monitored after CLP (Fig. 1a). Coincidentally, the survival rate was substantially higher in Ripk $^{-1-}$ Gsdmd $^{-1-}$ mice as compared with $R_{i p k 3^{-1-}}$ or $G s d m d^{-1-}$ mice, which also displayed significantly prolonged survival than WT mice (Fig. 1b). The serum levels of organ injury and cell damage indicators, including creatine kinase (CK), alanine aminotransferase (ALT), lactate dehydrogenase (LDH), HMGB1, and blood urea nitrogen (BUN) in WT mice were significantly reduced in Ripk $3^{-1-}$ and Gsdmd $d^{-1-}$

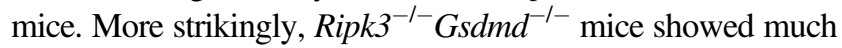
lower serum levels of these indicators compared with those in Ripk $^{-1-}$ or Gsdmd ${ }^{-1-}$ mice (Figs. 1c-f and S4c). Furthermore, we also analyzed the serum levels of cytokines. Ripk 3 or Gsdmd deficiency decreased IL- $1 \beta$ level from the early time point of sepsis (Fig. 1g), but reduced TNF $\alpha$, IFN $\beta$, and IL-6 production only at the late stage (Fig. 1h-j). Ripk $3^{-1-}$ Gsdmd ${ }^{-1-}$ mice exhibited the strongest protection against cytokine production. Notably, we also tested the effect of deficiency of both Mlkl and Gsdmd in this model. Consistently, $\mathrm{Mlkl}^{-1-} \mathrm{Gs} d m \mathrm{~d}^{-1-}$ mice were better protected compared with $\mathrm{Mlkl}^{-1-}$ or $G s d m d^{-1-}$ mice (Fig. S1 a-l). In addition, in TNF $\alpha$-induced lethality model, in line with previous study [18, 19], Ripk $3^{-l-}$ mice showed strong protection. Surprisingly, Gsdmd ${ }^{-1-}$ mice showed comparable protection as $R i p k 3^{-l-}$ mice against TNF $\alpha$-induced lethality. More strikingly, Ripk $3^{-1-}$ Gsdmd ${ }^{-1-}$ mice were completely resistant to TNFo-induced lethality (Fig. S2a). Similarly, WT mice succumbed to $20 \mathrm{mg} / \mathrm{ml}$ LPS challenge whereas $G s d m d^{-/-}$ mice tolerated significantly well to this lethal dose of LPS (Fig. S2b). And deficiency of both Ripk3 and Gsdmd led to much stronger protective effect against LPS-induced lethality (Fig. S2b). Taken together, these results suggested that both RIPK3-MLKL-mediated necroptosis and GSDMD-mediated pyroptosis contributed critically to sepsis-mediated pathologies and mortality.

\section{Deficiency of both Ripk3 and Gsdmd attenuates the mobilization and infiltration of inflammatory cells during sepsis}

It has been known that both RIPK3 and GSDMD contributed to the release of chemokines and cytokines including IL- $1 \beta$ and CXCL2 to enhance macrophage and neutrophil mobilization and infiltration [20, 27, 28]. Consistently, we found that the elevated levels of chemokines, especially for CXCL2 and CCL3 (Fig. 2a, b), were mostsignificantly reduced at both early and late stages of CLP-induced sepsis in Ripk $3^{-1-}$ Gsdmd $^{-1-}$ mice. To determine whether reduced production of chemokines in Ripk $^{-/-} \mathrm{Gsdmd}^{-1-}$ mice could lead decreased inflammatory cells recruitment, we examined the number of neutrophils and monocytes in the peripheral blood, lung, liver, and kidney after CLP surgery. Unexpectedly, fewest peripheral blood neutrophils and monocytes were detected in WT mice compared with those in knockout mice. Significantly increased neutrophils and monocytes were detected in the blood of Ripk $3^{-1-}$ Gsdmd $^{-1-}$ mice compared with that of 
a

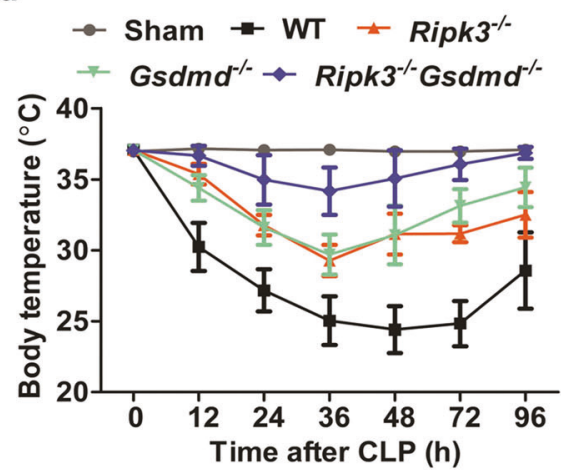

b

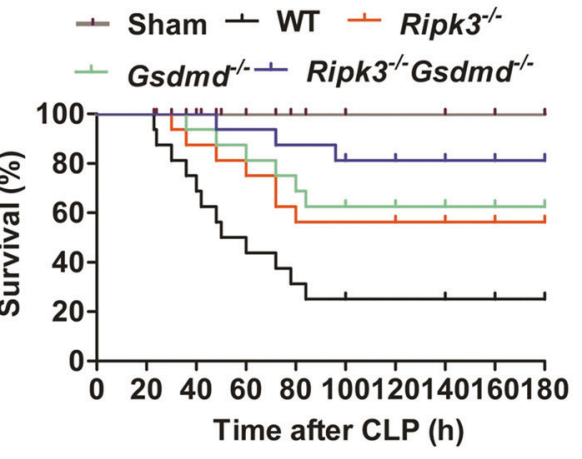

c

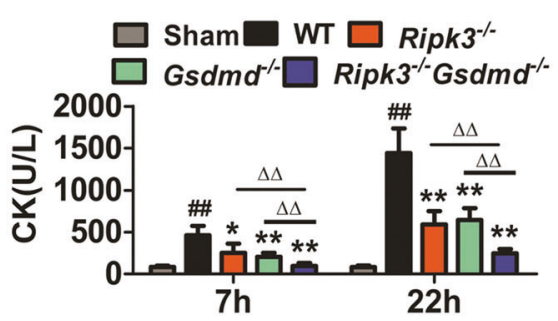

f

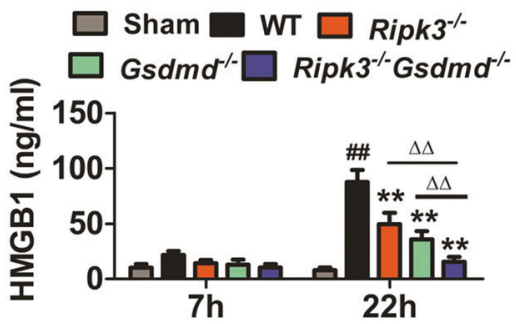

d

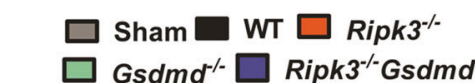

e

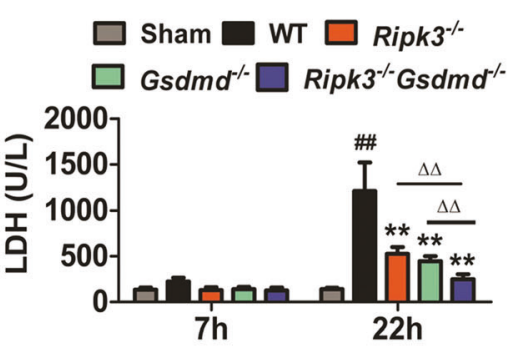

g

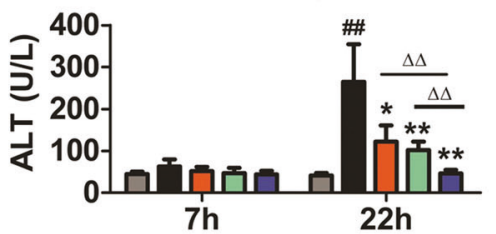

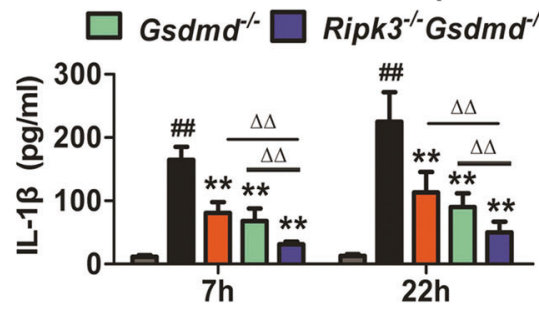

h

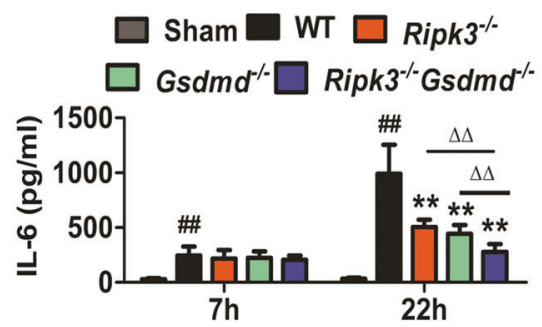

i

j
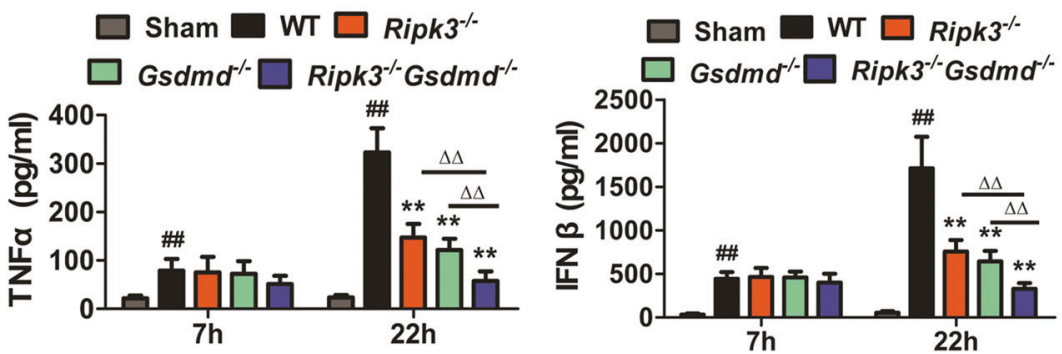

Fig. 1 RIPK3 and GSDMD are indispensable for CLP-induced

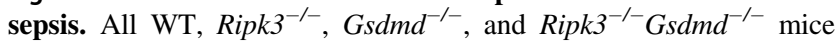
were subjected to severe CLP as described in "Methods". Sham operated mice underwent surgery without CLP. a Body temperature $(n=8)$ and $\mathbf{b}$ Survival $(n=16)$ were monitored as the indicated time. Another CLP experiment was performed and blood samples were

collected $(n=7)$ for the analysis of CK (c), ALT (d), LDH (e). HMGB1 (f) and cytokine production including IL-1 $\beta$, IL-6, TNF $\alpha$, $\operatorname{IFN} \beta(\mathbf{g}-\mathbf{j})$ were detected by ELISA $(n=6) .{ }^{\# \#} P<0.01$ vs Sham group; $* P<0.05$, $* * P<0.01$, vs WT group; ${ }^{\Delta} P<0.05,{ }^{\Delta \Delta} P<0.01$ vs Ripk3 $^{-/-} \mathrm{Gsdmd}^{-/-}$group.

Ripk3 $^{-/-}$or Gsdmd ${ }^{-/-}$mice (Fig. 2c, d). We next examined whether neutrophil spontaneous death was delayed by both Ripk3 and Gsdmd deficiency. Consistent with in vivo results, double deletion of Ripk3 and Gsdmd showed the most significantly delayed neutrophil spontaneous death while Ripk3 or Gsdmd deletion exhibited moderately delayed spontaneous cell death (Fig. 2e). In addition, comparable bacteremia was detected in WT and Ripk3 $3^{-1-}$

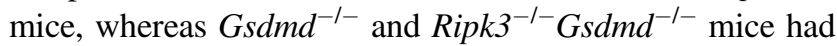
less severe bacteremia (Fig. 2f), indicating that mice with Gsdmd deficiency had a higher capacity to clear infection, consistent with the tendency of delayed neutrophil 
a

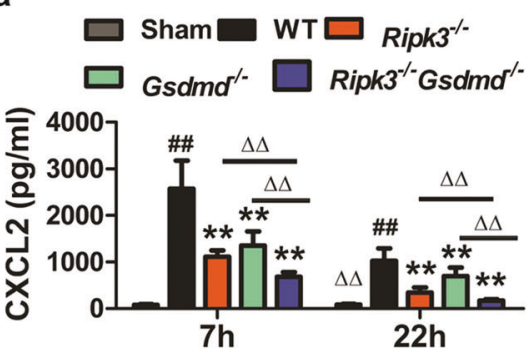

d

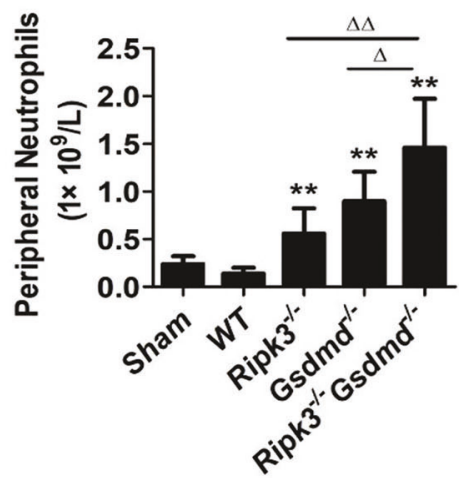

g

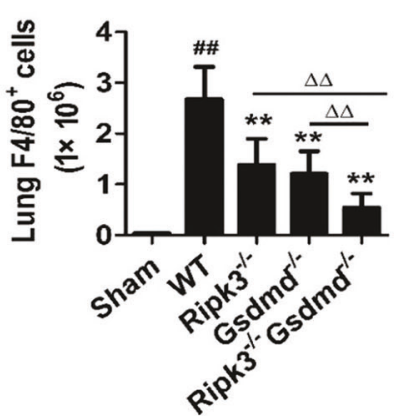

j

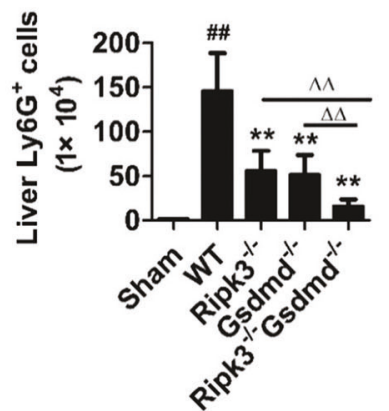

b

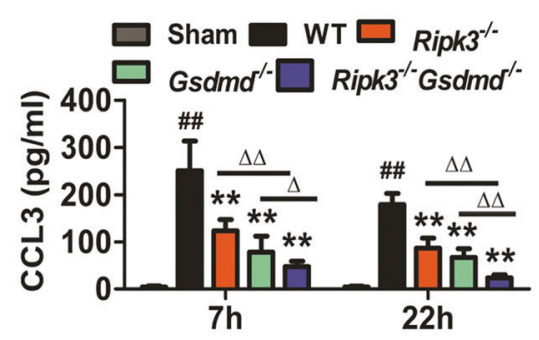

e

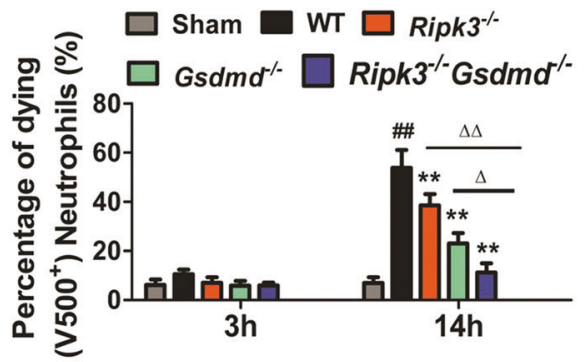

h
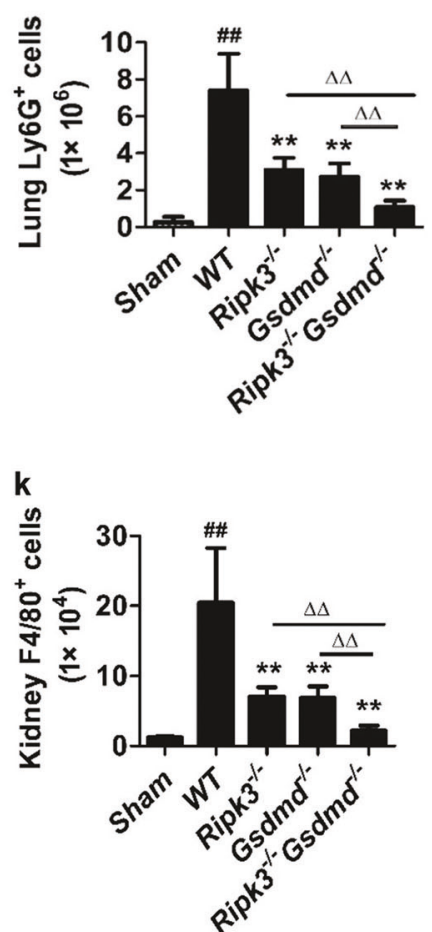

C
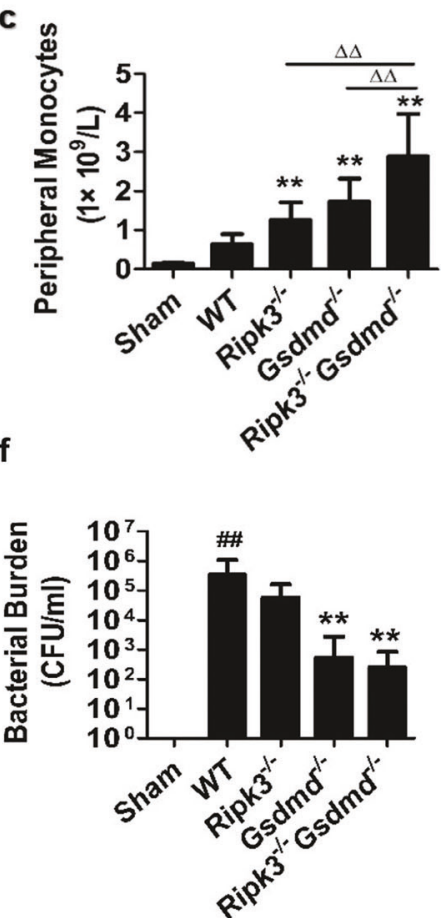

i
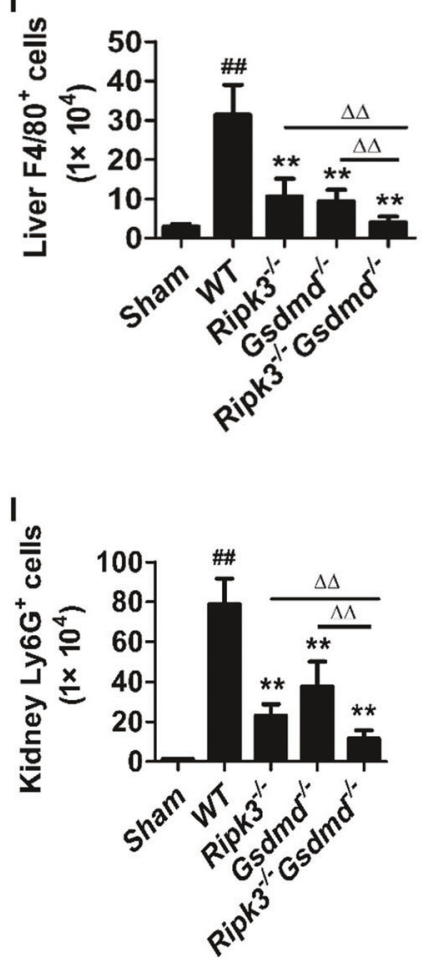

Fig. 2 Double deletion of Ripk3 and Gsdmd enhanced peripheral neutrophil and monocyte accumulation but reduced inflammatory cells recruitment to the tissue. All mice were treated as Fig 1. a, b Plasma chemokine levels including CCL3 and CXCL2 were evaluated by ELISA at 7 and $22 \mathrm{~h}$ after CLP. $n=6$. c, d Fresh blood samples were analyzed for total peripheral monocytes and neutrophils. $n=6$. e Peripheral blood neutrophils from WT, Ripk $3^{-/-}, G_{s} d m d^{-1-}$, and
Ripk ${ }^{-1-} \mathrm{Gsdmd}^{-1-}$ mice were purified and cultured. V500 positive indicated cell death. $n=5$. f Blood bacteria growth were compared. $n=6$. $\mathbf{g}-\mathbf{l}$ The number of macrophages $\left(\mathrm{F} 4 / 80^{+}\right)$or neutrophils $\left(\mathrm{Ly} 6 \mathrm{G}^{+}\right)$in lung, liver and kidney were detected by flow cytometry. $n=5$. ${ }^{\#} P<0.01$ vs Sham group; $* P<0.05, * * P<0.01$, vs WT group; ${ }^{\Delta} P<0.05,{ }^{\Delta \Lambda} P<0.01$ vs Ripk $3^{-/-}$Gsdmd ${ }^{-/}$group. 
spontaneous death. Interestingly, $\operatorname{Ripk}^{-{ }^{-1}} \mathrm{Gs} d m d^{-1-}$ mice displayed the highest reduction of neutrophil or monocyte infiltration into lung, liver, and kidney as detected by flow cytometry (Fig. 2g-1) and confirmed by histological examination (Fig. S3a-i). These results indicate that blockade of both necroptosis and pyroptosis improved neutrophil survival, resulting in enhanced clearance of bacteria and decreased infiltration of inflammatory cells into vital organs in septic mice.

\section{RIPK3 signaling collaborates with GSDMD to enhance sepsis-induced acute lung injury (ALI)}

It has been previously reported that CLP markedly increased pro-GSDMD and cleaved GSDMD protein levels in the lung tissue [29]. RIPK3 was rapidly increased in lung and plasma after administration of LPS [30]. Consistent with these studies, we found that CLP increased RIPK3 and pro- and cleaved GSDMD in lung tissue of WT mice (Fig. 3a), implicating the activation of both necroptosis and pyroptosis pathway in the CLP-induced sepsis. However, deletion of Ripk3 did not affect GSDMD activation and vice versa (Fig. 3a). Histology of lung tissue was further analyzed through H\&E staining. WT mice displayed severe pulmonary edema, hemorrhage and alveolar wall thickening at $22 \mathrm{~h}$ after CLP, while these were attenuated in $\operatorname{Ripk}^{-1-}, G s d m d^{-1-}$,

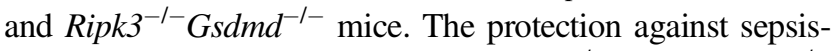
induced ALI was comparable in Ripk $3^{-1-}$ and $G s d m d^{-1-}$ mice while the strongest protection was observed in Ripk $3^{-/-}$ $G s d m d^{-1-}$ mice (Fig. 3b). Similar results were obtained in $M l k l^{-1-} G s d m d^{-1-}$ mice (Fig. S1c, d). Consistent with the histological changes, lung injury score (Fig. 3c), lung wet/dry weight ratio (Fig. 3d) and lung vascular permeability (Fig. 3e) were most significantly reduced in $R i p k 3^{-1-} \mathrm{Gsdmd}^{-1-}$ mice. ICAM-1 plays critical roles in mediating vascular leakiness and promoting the migration of immune cells $[31,32]$. In consistent with this, ICAM-1 expession was substantially increased in lung from WT group compared with that from sham group (Fig. 3f-h). Single knockout of Ripk3 or Gsdmd significantly reduced ICAM-1 expression whereas the double deficiency produced the greatest effect (Fig. $3 f-h$ ), accompanied by a similar pattern of change in the production of the inflammatory cytokines TNF $\alpha$ and IL-1 $\beta$ (Fig. 3i, j). These results indicate that combined blockade of necroptosis and pyroptosis provides better protective effect against CLPinduced ALI.

\section{Both necroptosis and pyroptosis are required for sepsis-induced intestine injury}

Pro-apoptotic caspase- 8 and propyroptotic caspase- 11 has been shown to collaborate for injury to the small intestine induced by systemic LPS challenge [33]. Whether there is a cooperation between pronecroptotic and propyroptotic signaling that may lead to bacterial sepsis-induced intestine damage remains poorly defined. We found that RIPK3/ MLKL phosphorylation, caspase-11 induction and GSDMD cleavage were prominent in the small intestine of CLP group compared with the sham group (Fig. 4a-c). Small intestinal tissues from WT mice displayed atrophy marked by sloughed and shed cells with condensed nuclei (Fig. 4d, f) and higher percentage of TUNEL staining-positive cells (Fig. 4e, g), whereas such intestinal damage was ameliorated in the Ripk $3^{-1-}$ or Gsdmd $d^{-1-}$ mice. The effect was strongest in the Ripk $^{-1-} \mathrm{Gsdmd}^{-1-}$ mice. Similar protection effect was obtained in $\mathrm{Mlkl}^{-1-} \mathrm{Gs} d \mathrm{md}^{-1-}$ mice (Fig. S1e, f). Likewise, intestinal leakiness evidenced by FITC-dextran flux which reflects the disruption of gut epithelial junction integrity was diminished to a greater extent in Ripk $3^{-/-}$

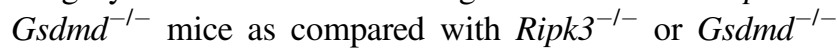
mice (Fig. 4h). These data suggest that both necroptosis and pyroptosis pathway contribute to inflammatory intestinal injury induced by bacterial sepsis.

\section{Both RIPK3 and GSDMD contribute to sepsis-induced systemic coagulation and liver and kidney injury}

Systemic coagulation such as disseminated intravascular coagulation is a grave pathologic state that often accompanies sepsis and results in microvessel occlusion, organ dysfunction, and lethality [34]. Therefore, we next investigated whether RIPK3 and GSDMD play roles in systemic activation of coagulation induced by CLP. As expected, three major indicators of hypercoagulability including the TF activity, plasma fibrinogen and D-dimer were all markedly elevated in the plasma of septic WT mice (Fig. 5a-c). In contrast, this effect was lessened to comparable extent by deficiency of either Ripk3 or Gsdmd. More strikingly, Ripk $3^{-1-}$ Gs $d m d^{-1-}$ mice showed much lower systemic activation of coagulation in CLPinduced sepsis. Consistent with these data, significant increase of blood vessels clotting was observed in the liver of WT mice compared with that in sham controls. Deletion of either Ripk3 or Gsdmd similarly diminished this effect, while double deletion of Ripk3 and Gsdmd completely inhibited blood vessels clotting (Fig. 5d, e). Similar results were obtained for fibrinogen deposition in the liver as assessed by immunostaining and immunobloting (Fig. 5f, g). In addition, although the liver (Figs. 1d, 5d, e) and kidney injury (Fig. S4a-c) in this CLP model were not very severe, ablation of Ripk3 or Gsdmd alone still showed partial protection and double deletion of Ripk3 and Gsdmd almost completely prevented the injury. Notably, $M l k l^{-/} G s d m d^{-/-}$mice also showed lower level of TF activity, ALT and BUN, the 


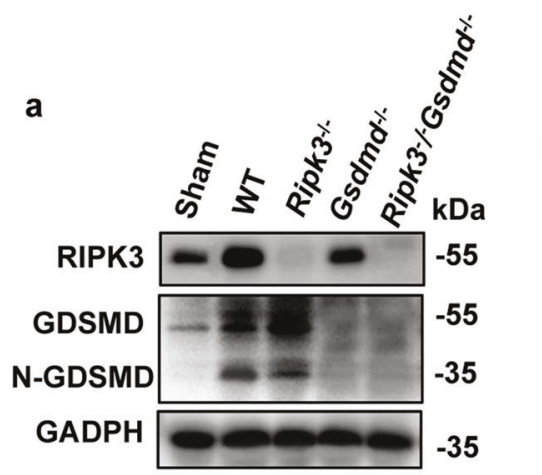

C

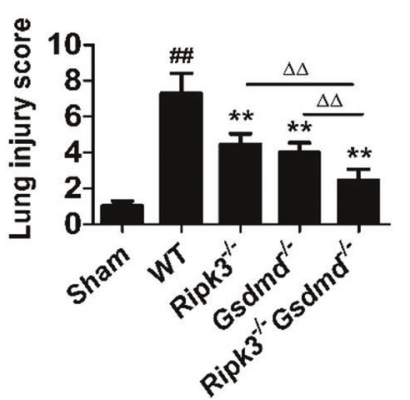

$\mathbf{f}$

ICAM-1

\section{Sham}

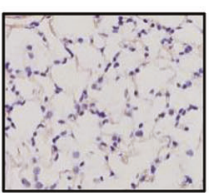

g ICAM-1

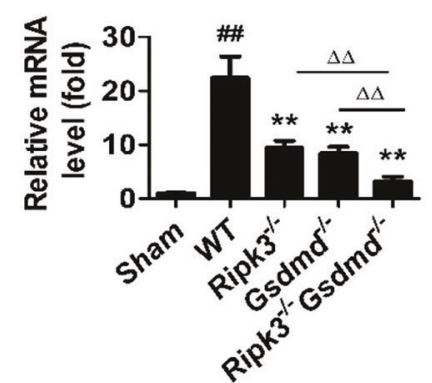

i

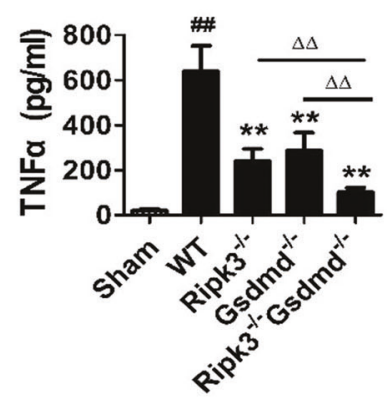

Fig. 3 Effects of RIPK3 and GSDMD on sepsis-induced lung injury. a Levels of RIPK3, GSDMD and cleaved-GSDMD increased $12 \mathrm{~h}$ after CLP by Western blot analysis. GAPDH was used as the loading control. $n=4$. b-j Mice were subjected to CLP for $22 \mathrm{~h} . n=$ 6. b Representative images of HE stained sections. Scale bar $=100$ $\mu \mathrm{m}$. c Histological scoring of lung injury. At least ten randomly selected fields were counted per mice. d lung Wet/Dry weight ratio for evaluating lung edema. e Mice were injected with Evans blue dye after

h
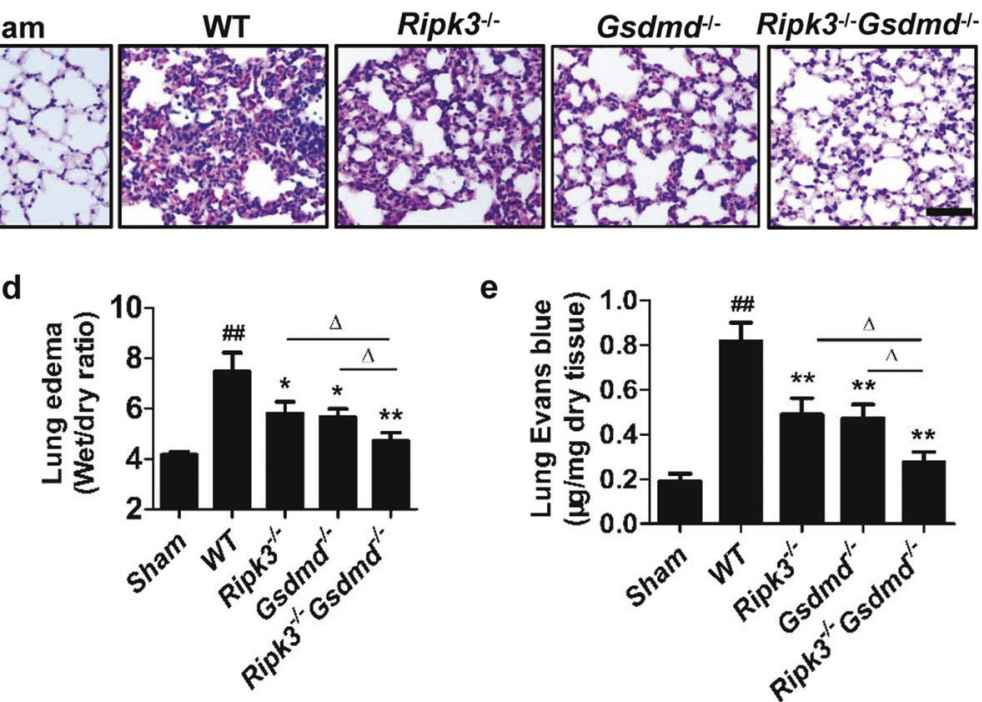

e

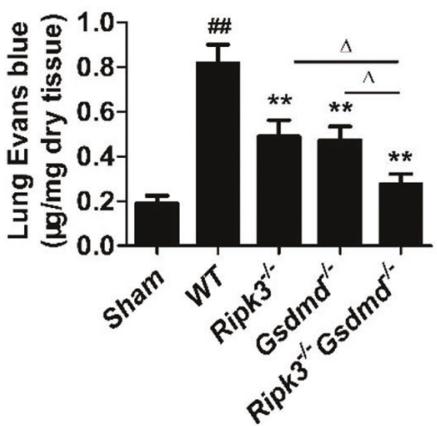

Ripk3-1-

Gsdmd ${ }^{-/-} \quad$ Ripk3 $^{-1-G s d m d^{-/-}}$
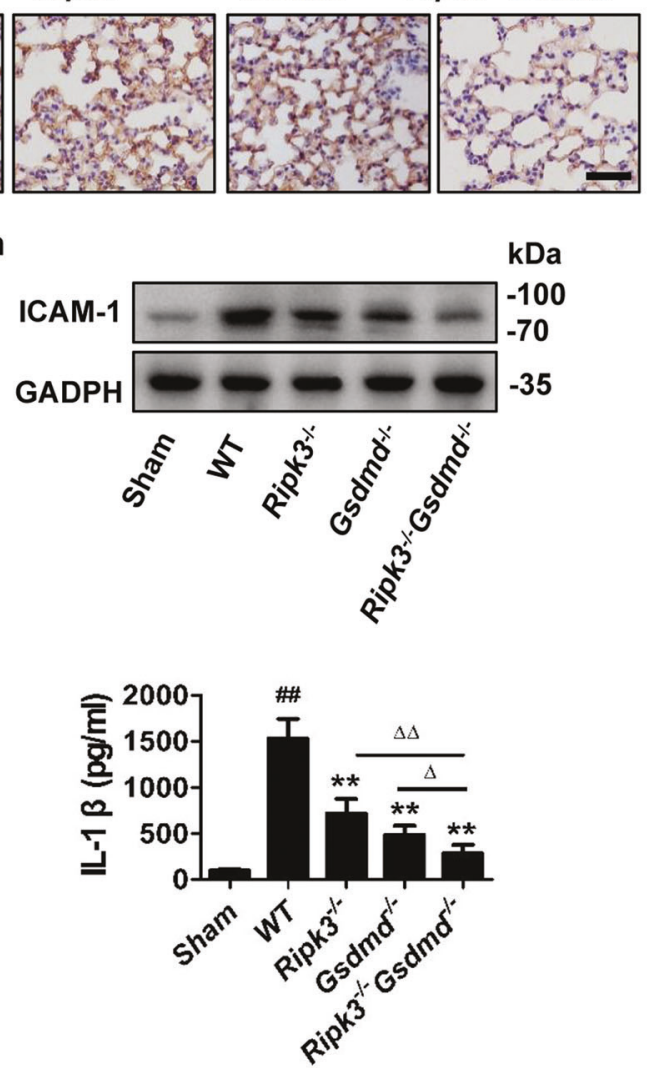

CLP, and spectrophotometry was used to measure the amount of dye extracted from the lung. f Representative images of immunohistochemistry of lung tissues with ICAM staining. Scale bar $=100 \mu \mathrm{m}$. g, h Quantitative RT-PCR and Western blot analysis for ICAM. i, j ELISA for TNF $\alpha$ and IL- $1 \beta .{ }^{\# \#} P<0.01$ vs Sham group; $* P<0.05$, ** $P<0.01$, vs WT group; ${ }^{\Delta} P<0.05,{ }^{\Delta \Delta} P<0.01$ vs Ripk $3^{-1-} G s d m d^{-1-}$ group. 
a

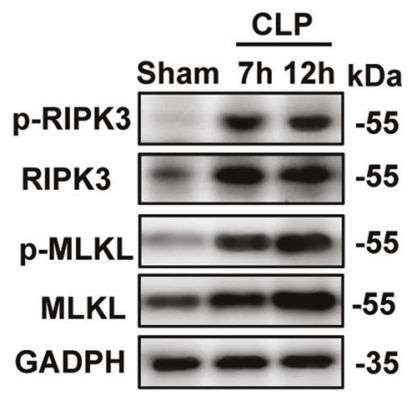

b

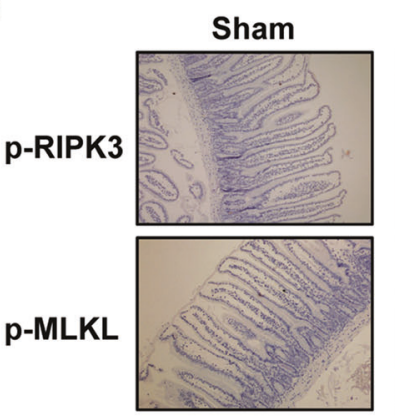

CLP
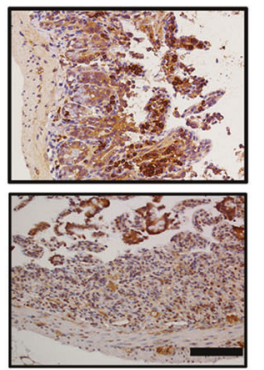

C

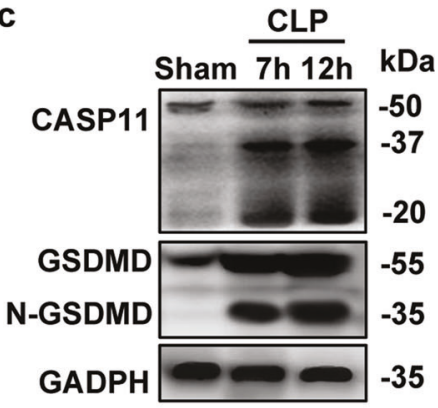

d
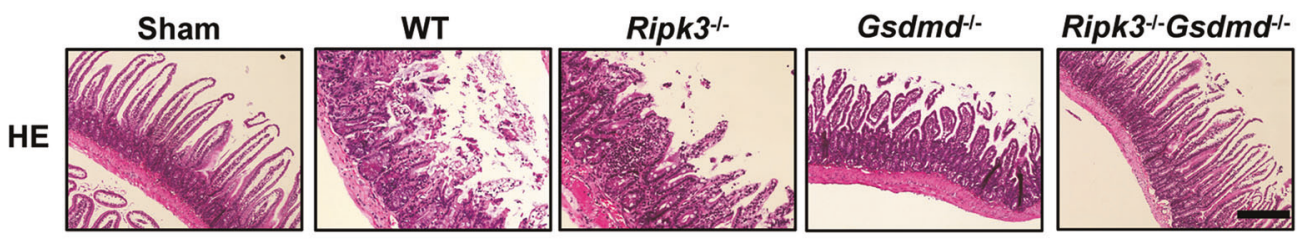

e
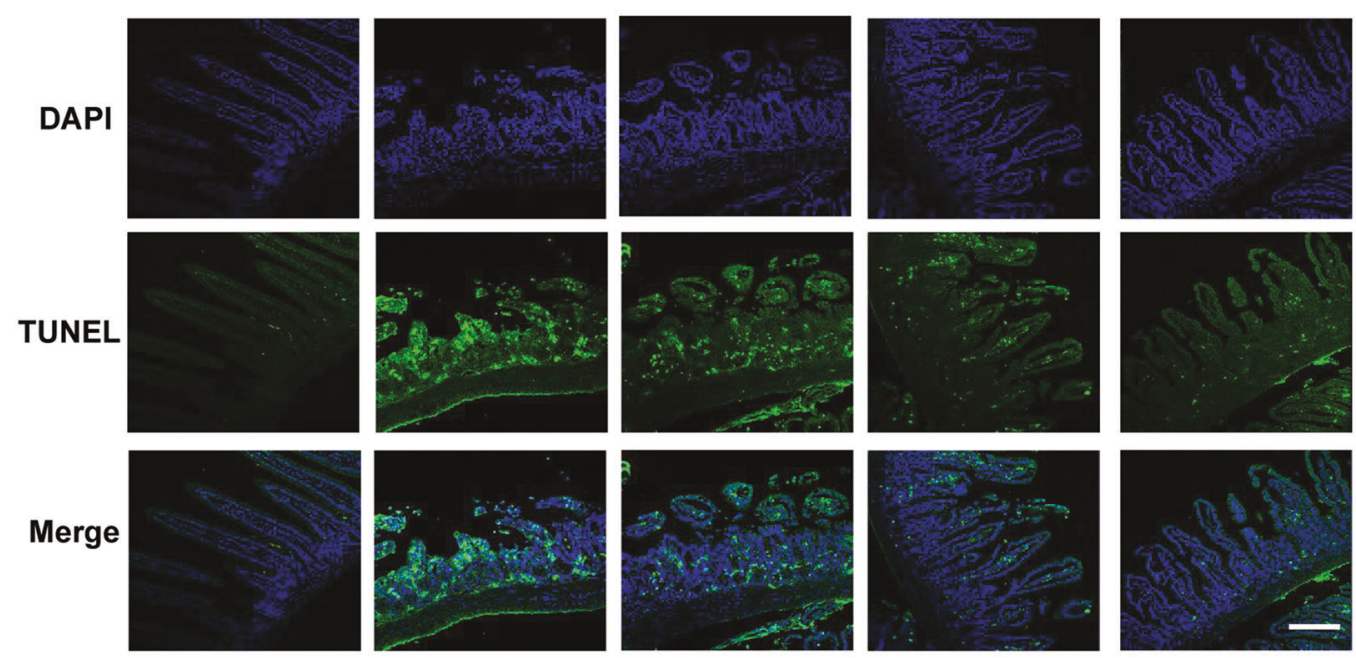

f

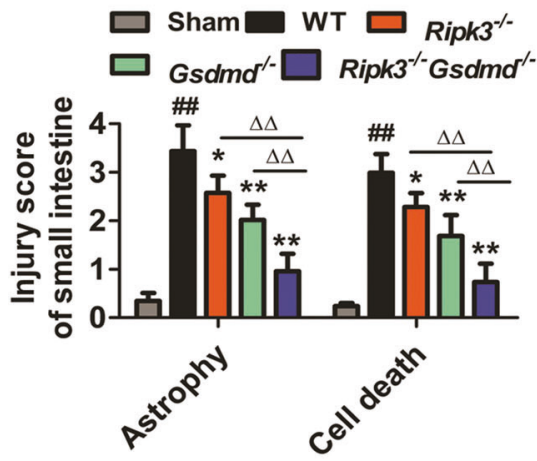

Fig. 4 Effects of RIPK3 and GSDMD on sepsis-induced intestine injury. The mice were subjected to severe CLP for the indicated hours. $n=6$. a Western blot analysis and RIPK3, p-RIPK3, MLKL, pMLKL in the intestines of mice after CLP. $n=4$. b Immunohistochemical staining of $\mathrm{p}$-RIPK3 and p-MLKL. Scar bar $=100 \mu \mathrm{m}$. c Detection of caspase-11 activation $(43,38,25 \mathrm{kDa})$ and Gsdmd cleavage in the intestines of mice after CLP. $n=4 . \mathbf{d}, \mathbf{f}$ Representative images of HE stained sections. Scale bar $=100 \mu \mathrm{m}$. Histological scoring of intestine atrophy and sloughed cells with nuclei damage

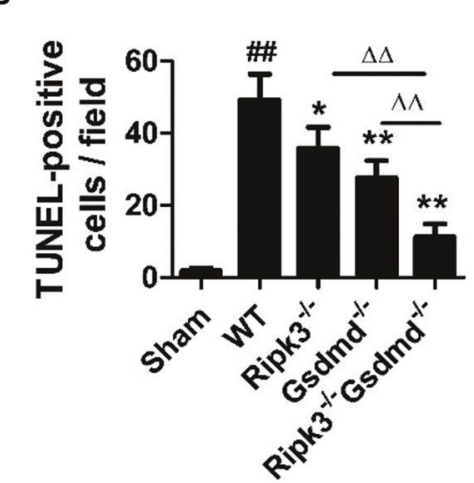

h

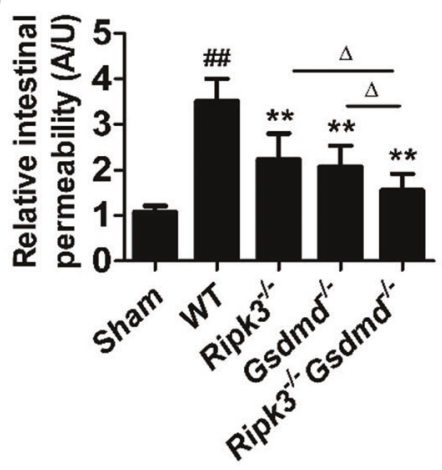

which indicating cell death are shown in $\mathbf{f} . n=6$. e, $\mathbf{g}$ Representative images of TUNEL staining for cell death examination are shown. TUNEL, green overlap with DAPI, blue. The original image is magnified $\times 100$. Scale bar $=100 \mu \mathrm{m}$. TUNEL-positive cells in ten randomly selected fields were counted per mice. $n=6$. $\mathbf{h}$ The levels of FITC-dextran in plasma was measured by fluorometry to indicate intestinal permeability. $n=6 .{ }^{\# \#} P<0.01$ vs Sham group; $* P<0.05$, ${ }^{*} P<0.01$, vs WT group; ${ }^{\Delta} P<0.05,{ }^{\Delta \Delta} P<0.01$ vs Ripk $3^{-/-} G s d m d^{-/-}$ group. 
a

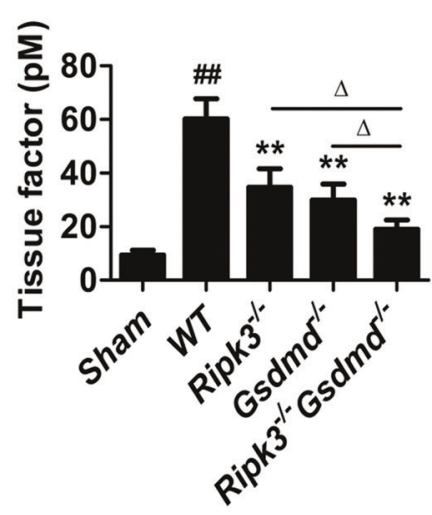

b

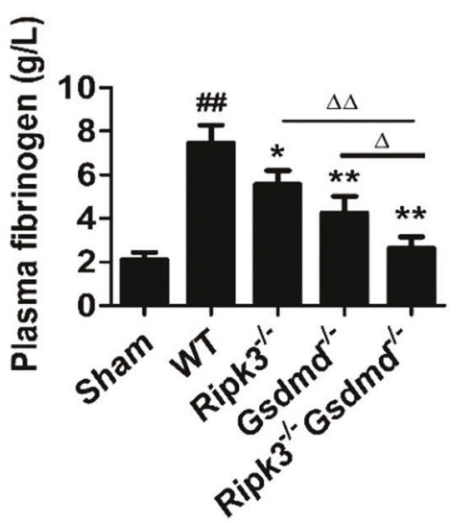

C

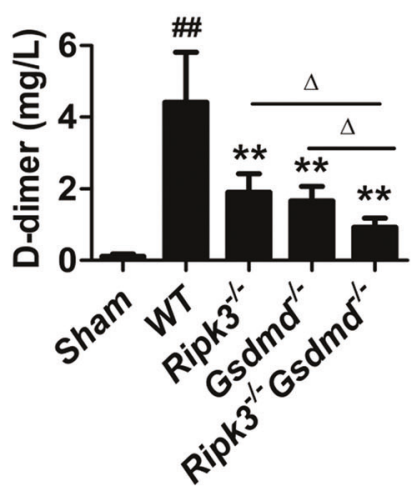

d

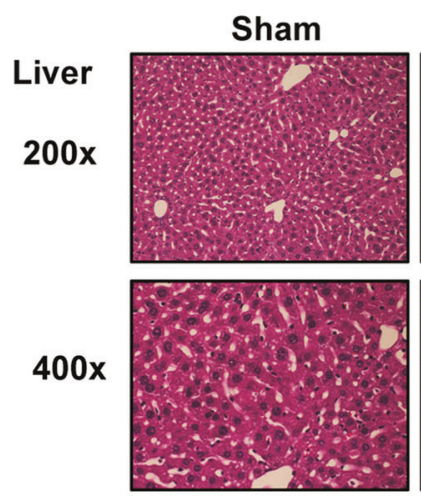

e

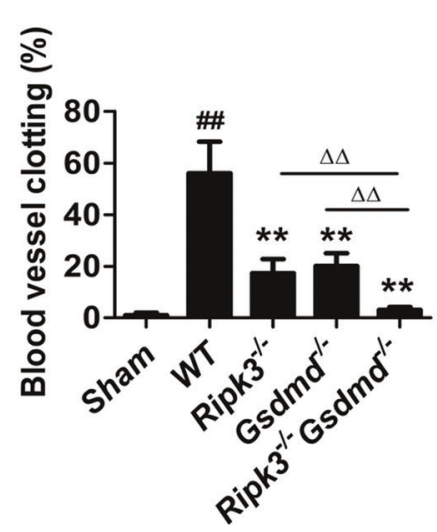

WT
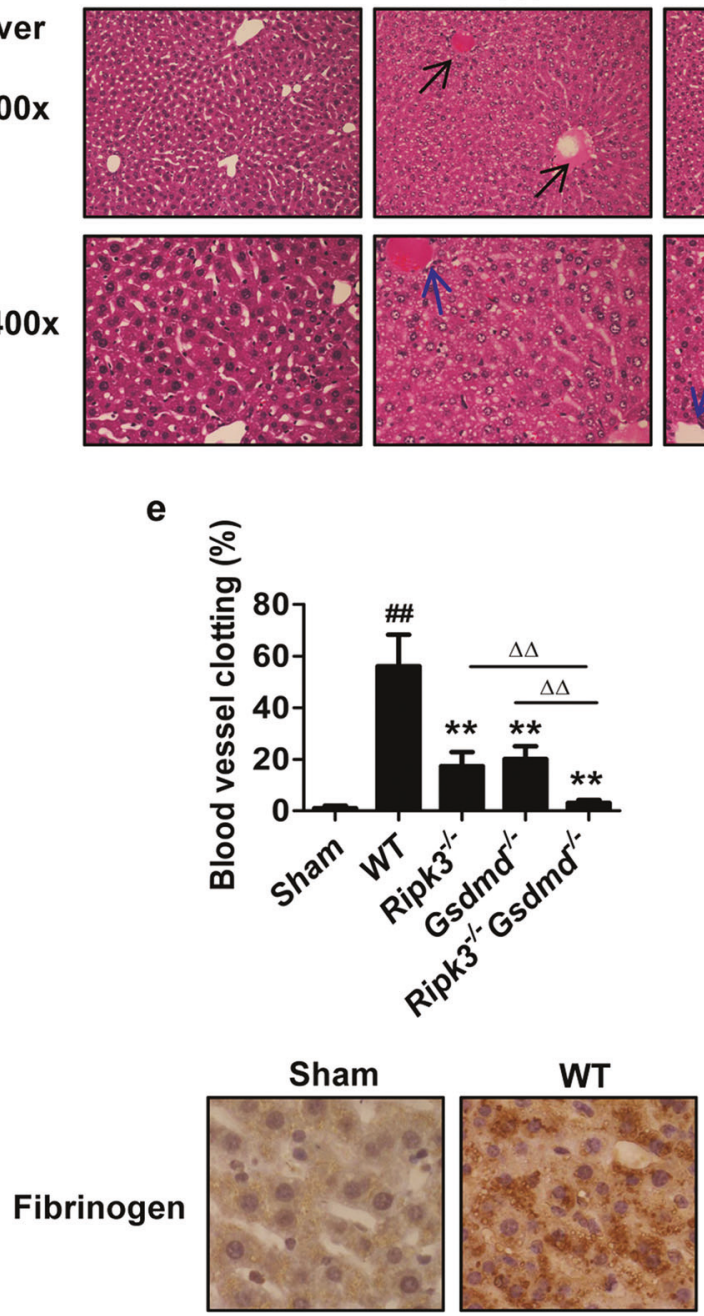

Fig. 5 Effects of RIPK3 and GSDMD on sepsis-induced systemic coagulation and liver injury. The mice were subjected to severe CLP for $22 \mathrm{~h} . n=6$. Blood were collected for measuring TF activity level (a) and the concentration of plasma fibrinogen (b) and D-dimer (c). d Mice were euthanized and perfused with PBS, then fixed with $4 \%$ formalin using perfusion fixation. Black arrows indicated clotting of blood vessels (Scale bar $=100 \mu \mathrm{m}$ ) and blue arrows indicated inflammatory cells (Scale bar $=50 \mu \mathrm{m}$ ) in representative images of H\&E-stained liver sections. The liver sections in WT mice showed that
Ripk3-1-
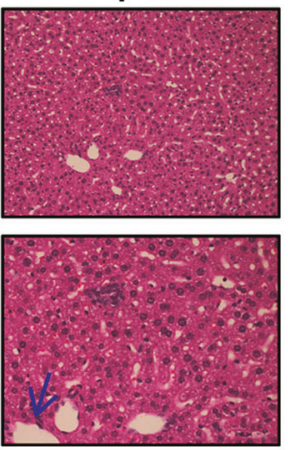

f

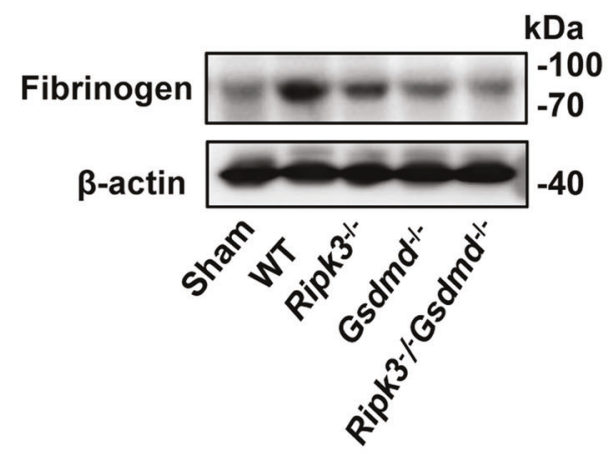

Ripk $3^{-1-}$
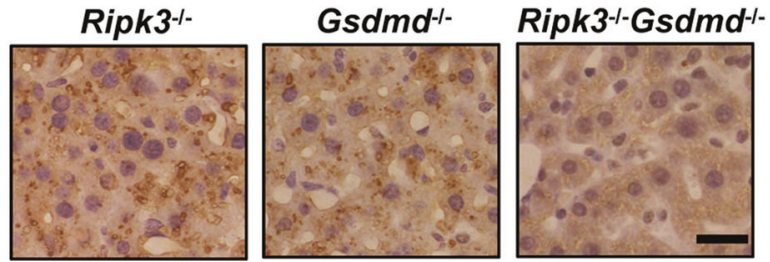

cell swelling, focal necrosis and inflammatory cell infiltration were significantly attenuated by ablation of Ripk3 or Gsdmd alone with the greater effect when both Ripk3 and Gsdmd were knocked out. e Quantification of clotting observed in liver blood vessels. f Representative immunoblot for fibrinogen by Western blot. $g$ Liver sections were immunostained with the antifibrinogen antibody. Representative sections are shown. ${ }^{\# \#} P<0.01$ vs Sham group; $* P<0.05$, $* * P<0.01$, vs WT group; ${ }^{\Delta} P<0.05,{ }^{\Delta \Delta} P<0.01$ vs Ripk $3^{-/-}$Gsdmd ${ }^{-\prime-}$ group. 
indicators of hypercoagulability and liver and kidney function, compared with $M l k l^{-/-}$or $G s d m d^{-/-}$mice (Fig. S1g, h, j). Thus, combined blockade of necroptosis and pyroptosis contributes to better prevention of systemic coagulation and organ injury to liver and kidney triggered by the lethal sepsis.

\section{Double deletion of Ripk3 and Gsdmd in myeloid and nonmyeloid cells protects against sepsis-induced organ damage}

To determine the contributions of BM-derived inflammatory cells and tissue intrinsic cells to the sepsis-induced pathogenesis, we performed BM transplantation studies. Ripk $^{+/+} \mathrm{Gsdmd}^{+/+}$(WT) to $\operatorname{Ripk}^{+/+} \mathrm{Gsdmd}^{+/+}$(WT) chimeric mice had a high mortality rate (Fig. 6a), high levels of inflammatory cytokines TNF $\alpha$, IFN- $\beta$, IL1 $\beta$ (Fig. 6b-d), and TF release (Fig. 6k), as well as severe lung (Fig. 6e, f) and intestine (Fig. 6e, g) injury, and moderate kidney (Fig. 6e, h, i) and liver (Fig. 6e, j) injury. However, these characteristic manifestations were significantly attenuated in $R i p k 3^{+/+}{ }^{-1 s} d m d^{+/+}$(WT) to $R i p k 3^{-/} G s d m d^{-/-}$ (GR), Ripk3 ${ }^{-/-}$Gsdmd $^{-/-}$(GR) to Ripk ${ }^{+/+}$Gsdmd $d^{+/+}$ (WT), Ripk ${ }^{-/-}$Gsdmd $^{-/-}$(GR) to Ripk ${ }^{-/-}$Gsdmd ${ }^{-1-}$

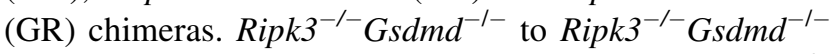
chimeras revealed the strongest protection. Ripk $3^{-1-}$ Gsdmd $^{-/-}$to Ripk ${ }^{+/+} G s d m d^{+/+}$reduced cytokines production more significantly than $R i p k 3^{+/+} G s d m d^{+/+}$to

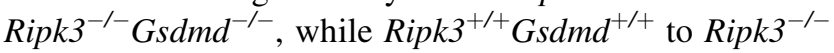
Gsdmd $d^{-1-}$ exhibited better organ protection than Ripk3 ${ }^{-/-}$ $G s d m d^{-/-}$to $R i p k 3^{+/+} G s d m d^{+/+}$, indicating that nonmyeloid necroptosis and pyroptosis, likely triggered by proinflammatory cytokines, critically contribute to sepsisinduced tissue injury. These results suggest that necroptosis and pyroptosis in both myeloid and nonmyeloid cells critically contribute to the progression of sepsis and tissue nonmyeloid cells may play a more important role in sepsisinduced tissue injury.

\section{Ablation of both Ripk3 and Gsdmd inhibits necroptotic and pyroptotic stimuli-induced IL-1 $\beta$ and TF release in BMDMs}

Given that Ripk3/Gsdmd double deficiency renders better protection against the CLP sepsis-induced tissue damage and systemic coagulation, we were interested to understand how necroptosis vs pyroptosis correlated with cell death, inflammatory cytokine secretion and TF release triggered by inflammatory cells. BMDMs were obtained from the relevant gene targeted mice. In these isolated BMDMs, necroptosis or pyroptosis was triggered according to previous studies [3538]. Cell death and IL- $1 \beta$ activation triggered by LPS/QVD/ Smac or $\mathrm{TNF} \alpha / \mathrm{CHX} / \mathrm{Smac}$ were entirely prevented by Ripk3 deficiency or Ripk3/Gsdmd or Mlkl/Gsdmd double deficiency but not by Gsdmd deficiency alone (Figs. 7a, b, f and S5a, c, d). In contrast, nigericin-induced cell death and caspase-1 activation (Fig. 7a, b) as well as HMGB1/LPS-induced cytotoxicity and caspase-11 activation (Fig. 7c, d) were not affected by the absence of Ripk3, but was completely prevented wherever Gsdmd deficiency occurred. The extent of BMDM cell death was in parallel with the secretion of both IL-1 $\beta$ (Figs. 7b, d, and S5c, d) and TF (Figs. 7e, g and S5b). Thus, these results further support the idea that both pyroptotic and necroptotic macrophages facilitate the release of IL-1 $\beta$ and TF to drive inflammatory response and system coagulation during sepsis.

\section{Double deletion of Ripk3 and Gsdmd prevents necroptotic and pyroptotic stimuli-triggered cell cytotoxicity and TF activation in endothelial cells}

Endothelial cell death or dysfunction leads to increased vascular permeability and clotting, which contributes to sepsisinduced multiple-organ damage. It has been shown that both RIPK1-dependent EC necroptosis and bacterial endotoxin LPS-triggered EC pyroptosis contributed to vascular permeability, coagulation and organ damage [20, 39]. However, whether RIPK3 collaborates with GSDMD to execute EC death and consequently destroy endothelial barrier is yet unknown. Thus, we isolated ECs from the lung of mice, and exposed them to necroptotic or pyroptotic stimuli. As shown in Figs. 8a-c and S5e, f, RIPK3 and MLKL was required for TNFo-induced cell death as evidenced by enhanced cell viability in Ripk3 or Mlkl deficient cells, accompanied by a remarkable reduction of TF activity. On the other way around, GSDMD was responsible for LPS-induced cell death and TF release when the cells were treated with LPS plus HMGB1 or LPS transfection. To further confirm this specificity, we reconstituted RIPK3 expression in Ripk $3^{-/-}$cells (Fig. 8d) and found that reconstitution of RIPK3 sensitized the initially resistant cells to TNF $\alpha$-induced cytotoxicity (Fig. 8e). Similarly, when GSDMD was reconstituted in the Gsdmd $d^{-1-}$ cells (Fig. 8f), the reconstituted GSDMD was successfully cleaved to generate N-terminal fragment of GSDMD, which restored the sensitivity of cells to the cytotoxic effect of LPS (Fig. 8g). Translocation GSDMD-N to plasma membrane is required to trigger pyroptosis. A previous study showed that hormone-binding domain (HBD)mediated GSDMD dimerization can mimic stimulus-induced and GSDMD-mediated activation of pyroptosis [40]. As expected, induction of GSDMD-N-HBD interaction by 4OHT was very potent at killing the ECs with N-GSDMD translocation to the plasma membrane (Fig. 8h, i). These data together suggest that RIPK3 and GSDMD account for TNF $\alpha$ and LPS-induced EC death, respectively. Interestingly, TNF $\alpha$ plus CHX and Smac, or TNF $\alpha$ plus IFN $\beta$ enhanced EC 
a L WT to WT \& RG to WT

- WT to RG -- RG to RG

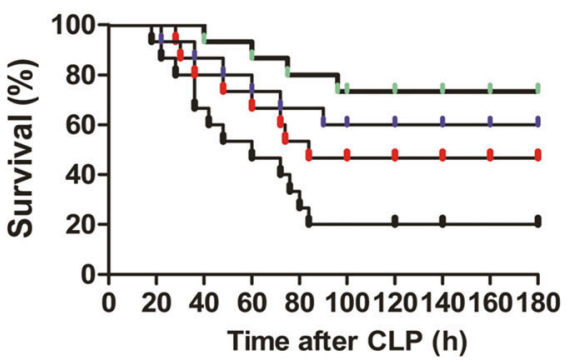

b

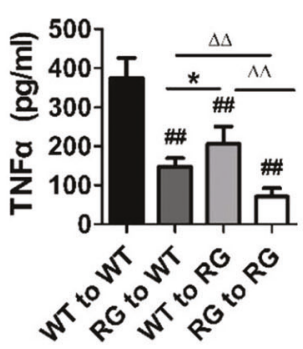

C

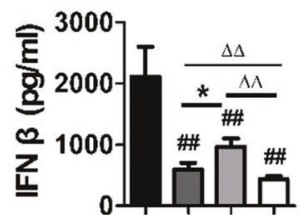

$2 \pi s_{0}$

250.2000 d

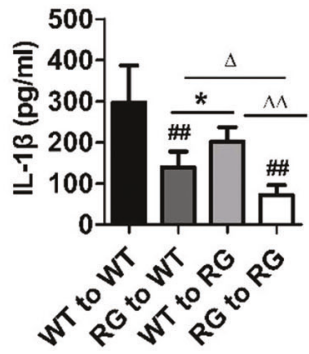

e
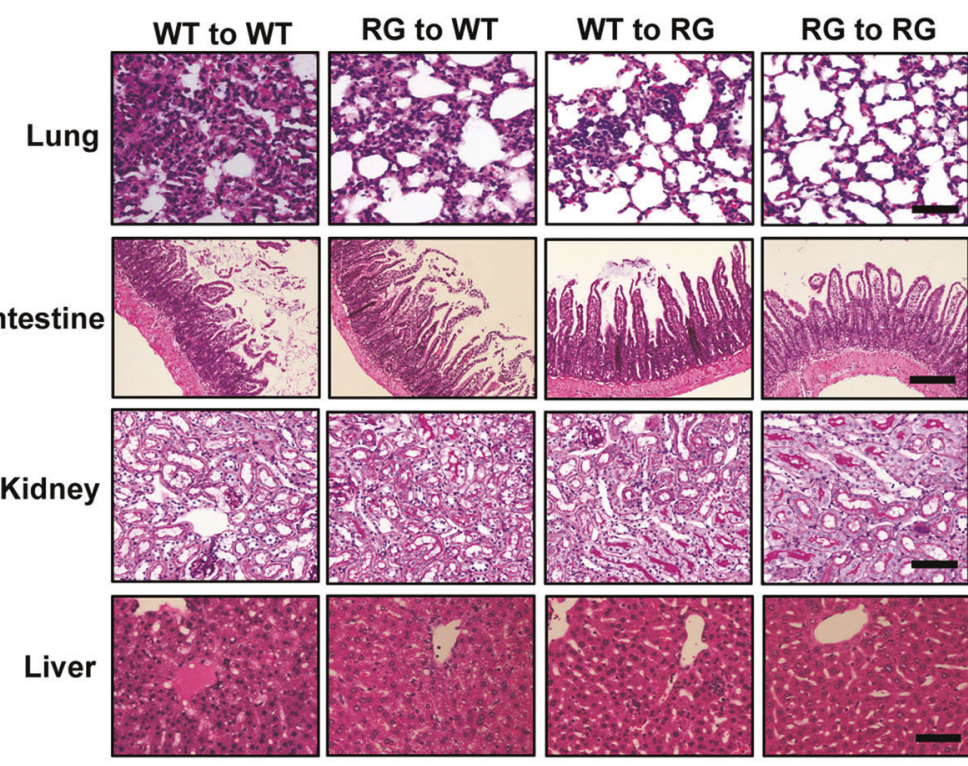

f
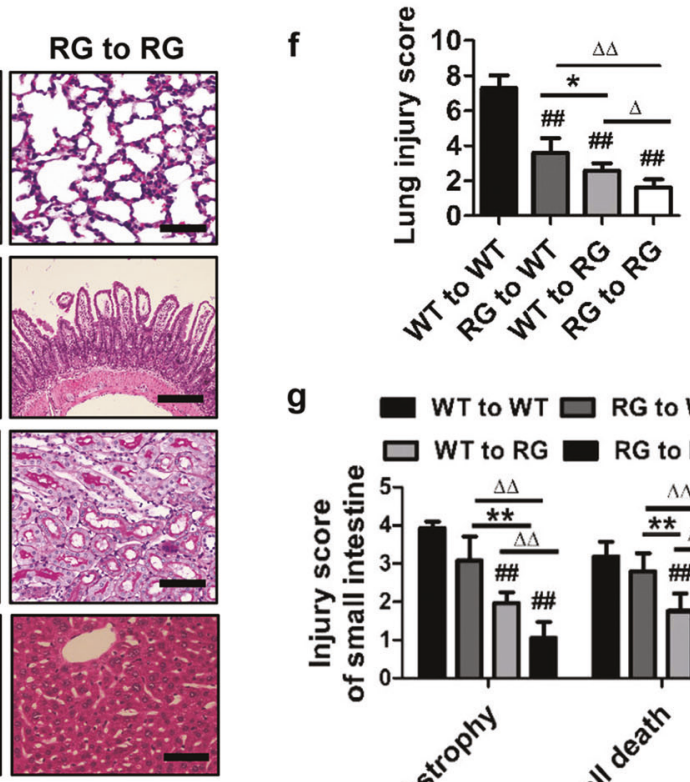

h

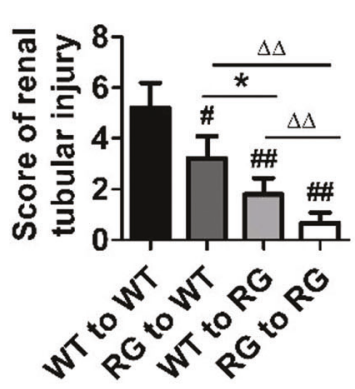

i

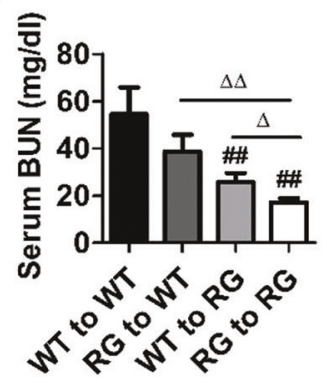

Fig. 6 RIPK3 and GSDMD in myeloid and nonmyeloid cells contributed to sepsis-induced organ damage. Chimeric mice bone marrow (BM) was replaced with donor BM cells from WT or from Ripk $^{-/-}$Gsdmd $^{-/-}(R G)$ mice. Irradiated WT and Ripk ${ }^{-/-}$Gsdmd ${ }^{-/-}$ mice were reconstituted with either WT or Ripk $3^{-1-}$ Gs $_{\text {Smd }}{ }^{-1-}$ BM cells. a Survival proportions as described. $n=12$. b-d Mice were challenged with CLP for $22 \mathrm{~h}$. TNF $\alpha$, IFN $\beta$, and IL- $1 \beta$ level were

permeability, which was attenuated by Ripk3 deficiency and Ripk3/Gsdmd double deficiency but not by Gsdmd deficiency (Fig. S6a, b). Medium from LPS-treated BMDMs could increase EC permeability, which was diminished in

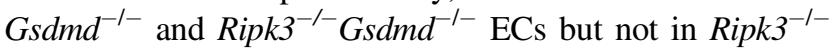

j

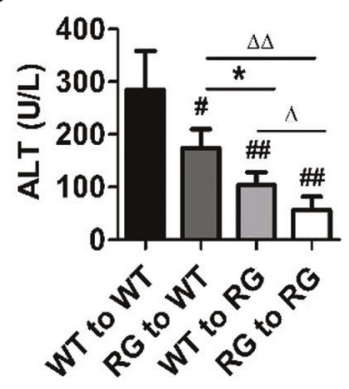

determined by ELISA. e Representative images of lung, intestine, liver, kidney of HE stained sections. Scar bar $=100 \mu \mathrm{m}$. f-h Histological scoring of lung, intestine and kidney injury. $\mathbf{i}, \mathbf{j}$ The biomarkers of kidney function (BUN) and liver function (ALT). $\mathbf{k}$ TF activity was evaluated. b-k $n=6$. RG: Ripk $3^{-/-}$Gsdmd ${ }^{-/-}$mice. ${ }^{\# \#} P<0.05$, ${ }^{\# \#} P<$ 0.01 vs WT to WT group; $* P<0.05, * * P<0.01$, vs WT to RG group; ${ }^{\Delta} P<0.05,{ }^{\Delta} P<0.01$ vs RG to RG group.

ECs (Fig. S6c). These results suggest that inflammatory cytokines produced by immune cells could promote the progression of EC injury, implicating a positive feedback loop involving inflammation and EC damage that ultimately contributed to sepsis progression. 
a

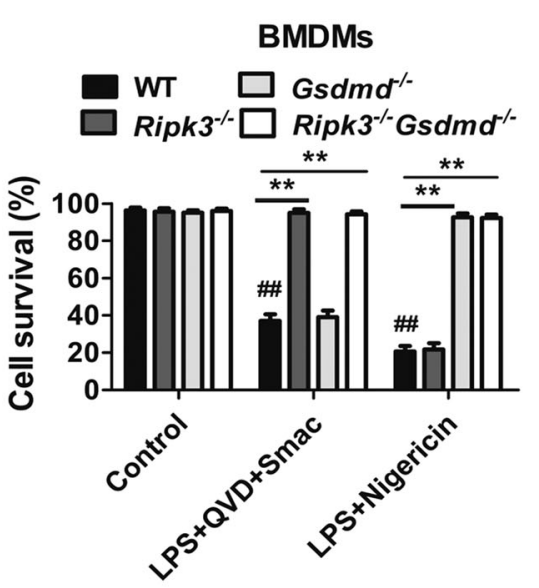

c

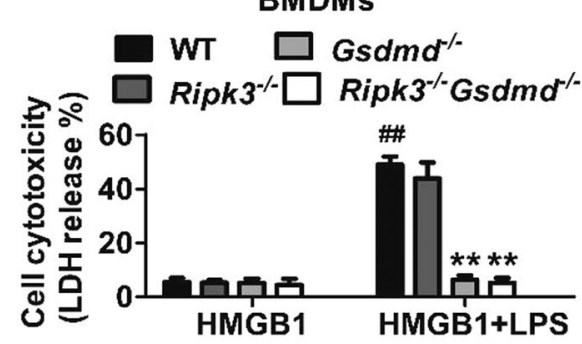

e

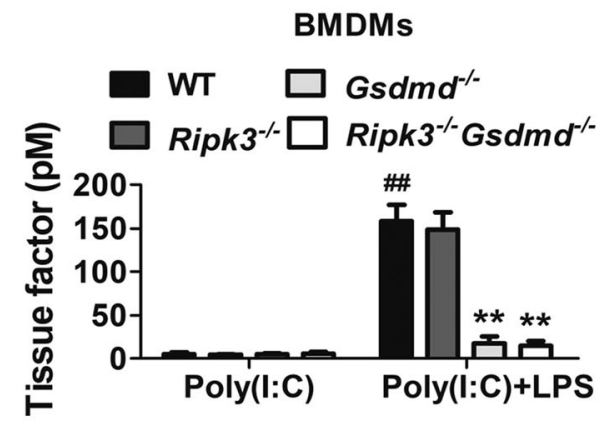

f

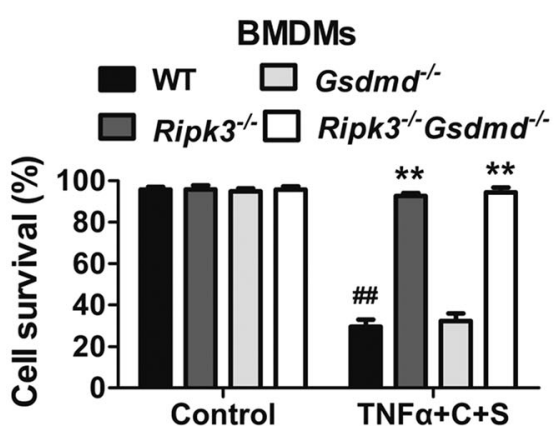

\section{Discussion}

The discoveries of RIPK3/MLKL-dependent necroptosis and GSDMD-dependent pyroptosis by genetic deletion brought new insights into the molecular mechanisms governing programmed necrotic cell death and inflammation activation. Recent studies demonstrated that individual deficiency of Ripk3 or Gsdmd conferred protection against

b

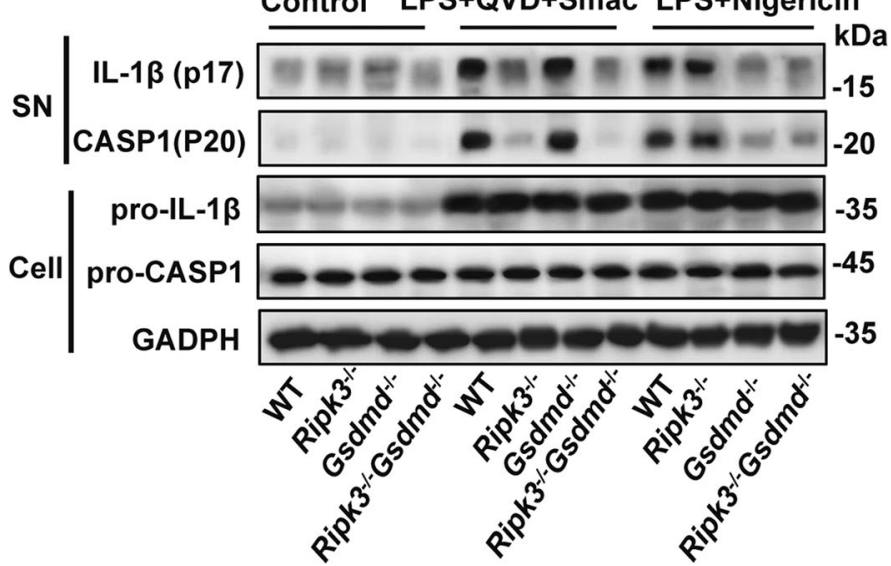

d

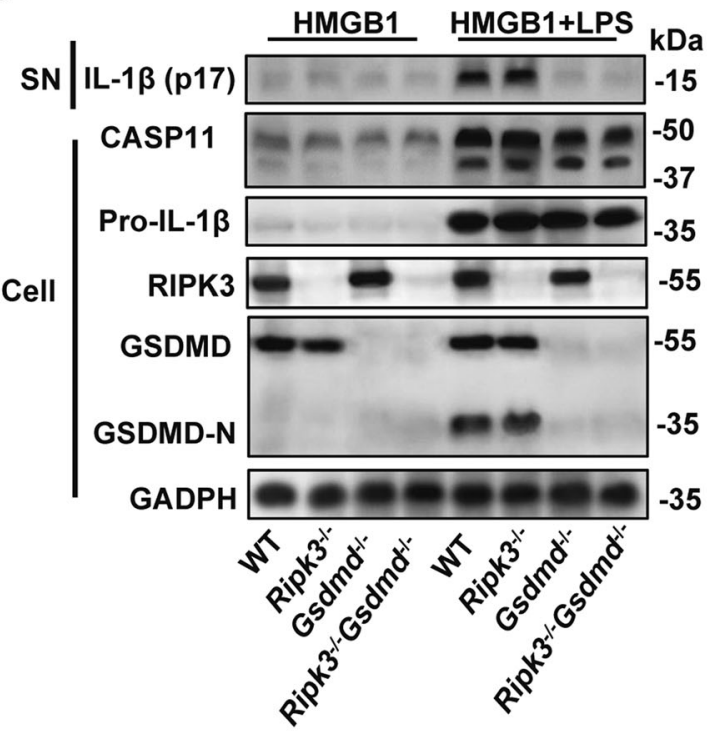

g

BMDMs

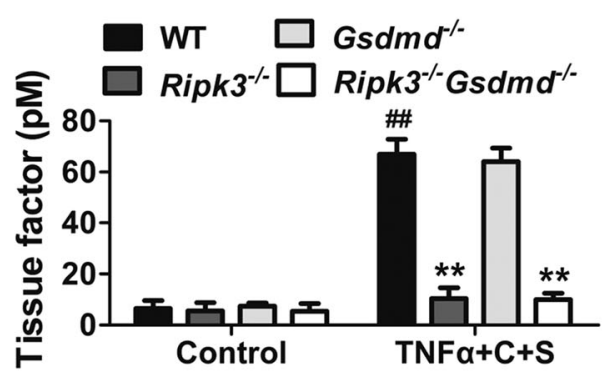

TNF $\alpha$-induced lethal SIRS or CLP-induced sepsis [18], or LPS-induced lethal septic shock [21]. Kinase-inactive RIPK1 mutant has also been shown to protect mice from TNF $\alpha$-induced lethal SIRS by preventing EC necroptosis [20]. However, it arose an important whether these individual necrotic cell death pathways are functionally or mechanistically interconnected. Therefore, in this study we set out to explore whether the combined loss of Ripk3 and 
Fig. 7 Double deletion of RIPK3 and GSDMD in BMDMs resisted both necroptotic and pyroptotic stimulation. BMDMs were

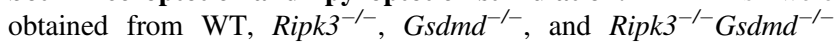
mice. $n=4$. a, b BMDMs were pretreated with LPS $(1 \mu \mathrm{g} / \mathrm{ml})$ for $3 \mathrm{~h}$, and then preincubated with Q-VD-OPh $(10 \mu \mathrm{M})$ for $30 \mathrm{~min}$ before stimulation with Smac mimetic $(10 \mu \mathrm{M})$ for $5 \mathrm{~h}$, or treated with $15 \mu \mathrm{M}$ Nigericin for $1 \mathrm{~h}$. Cell survival was determined by Propidium Iodide (PI) and Hoechst double-staining (a). Cell culture supernatants (SN) and total cell lysates were analyzed for IL- $1 \beta$ and Caspase 1 activation by western blot as indicated (b). ${ }^{\# \#} P<0.01$ vs Control WT group, ${ }^{* *} P$ $<0.01$ vs $\mathrm{LPS}+\mathrm{QVD}+$ Smac WT group or LPS + Nigericin WT group. c, d BMDMs were incubated with $2 \mu \mathrm{g} / \mathrm{ml}$ LPS and $500 \mathrm{ng} / \mathrm{ml}$ HMGB1 for $16 \mathrm{~h}$. LDH level were analyze for cell cytotoxicity. Total cell lysates were analyzed for pro-IL-1 $\beta$, caspase 11, GSDMD, and RIPK3. Culture supernatants (SN) were detected for IL-1 $\beta$ secretion. ${ }^{\# \#} P<0.01$ vs HMGB1 WT group, $* * P<0.01$ vs HMGB $1+$ LPS WT group. e For tissue factor (TF) activity evaluation, BMDMs were preincubated with $1 \mu \mathrm{g} / \mathrm{ml}$ Poly(I:C) for $5 \mathrm{~h}$, then transfected with PBS or $2 \mu \mathrm{g} / \mathrm{ml}$ LPS. ${ }^{\# \#} P<0.01$ vs Poly(I:C) WT group, $* * P<0.01$ vs Poly (I:C) + LPS WT group. f, g BMDMs were treated with $30 \mathrm{ng} / \mathrm{ml}$ $\mathrm{mTNF} \alpha$ plus $10 \mu \mathrm{g} / \mathrm{ml} \mathrm{CHX}$ in presence of $10 \mu \mathrm{M}$ SMAC-mimetic $(\mathrm{TNF} \alpha+\mathrm{T}+\mathrm{S})$ for $24 \mathrm{~h}$. ATP was measured for cell survival (f) and ELISA was used for TF activity detection $(\mathrm{g}) .{ }^{\# \#} P<0.01$ vs control WT group, $* * P<0.01$ vs TNF $\alpha+\mathrm{C}+\mathrm{S}$ WT group. All the Data are representative of three independent experiments.

Gsdmd could synergistically contribute to protection of multi-organ dysfunction during sepsis.

TNF $\alpha$-mediated necroptosis as an initial "cell death storm" could provoke acute or sustained inflammation. $\mathrm{TNF} \alpha$ is the direct and major proinflammatory cytokine that causes uncontrollable immune response and mortality during acute SIRS, the initial hyperinflammatory phase in sepsis [18]. LPS from Gram-negative bacterial pathogens is the classical endotoxin able to activate the host immune system leading to hyperinflammation, microcirculatory disturbance, and death [41]. Consistent with these previous studies, (i) we found that mice with Ripk3 or Gsdmd deficiency had significantly lower mortality treated with high dose of TNF $\alpha$; (ii) $G s d m d^{-1-}$ mice resisted significantly but not completely to $20 \mathrm{mg} / \mathrm{kg}$ LPS challenge and Ripk $3^{-/-}$ mice showed mildly resisted. Thus, our data showed that RIPK3 or GSDMD was not adequate to mediate TNF $\alpha$ and LPS-induced lethality. Moreover, we also found that double deletion of Ripk3 and Gsdmd conferred complete protection against TNF $\alpha$ and LPS-induced lethality. This promoted us to investigate how RIPK3 and GSDMD signaling collaborate to drive the progression of bacterial sepsis. We demonstrated that hypothermia and mortality in WT mice with the severe CLP condition were accompanied by the profound proinflammatory response. Double deletion of Ripk3/Gsdmd or Mlkl/Gsdmd showed the most significant protection. Interestingly, only IL-1 $\beta$ level but not IL-6, TNF $\alpha$ and IFN $\beta$ levels were reduced in mice with Gsdmd and/or Ripk3 deficiency at the early stage of sepsis. Consistent with some earlier reports, on the one hand, IL- $1 \beta$ can be released from the alive cells mainly dependent on the functionality of GSDMD and MLKL[27, 28, 42]; on the other hand, the production of cytokines IL- 6, TNF $\alpha$, and IFN $\beta$ at earlier stage during sepsis initiated by hematopoietic cells was independent on pronecroptotic and propyroptotic machinery [33]. The persistent increase of these cytokines in WT mice but not in Ripk3 or Mlkl and/or Gsdmd deletion mice should result from the consequence of DAMP release from necroptotic and pyroptotic cells at later stage of sepsis.

Ripk3 or Mlkl deficiency alone only moderately protected against the organ injury to lung, intestine, liver, kidney and inhibited blood coagulation in this CLP model of sepsis. Likewise, deficiency of Gsdmd alone afforded partial protection to the similar extent to Ripk3 or Mlkl single deficiency whereas double deletion of Ripk3/Gsdmd or Mlkl/Gsdmd provided the strongest protection. Of note, $G s d m d^{-/-}$mice appeared to have stronger capacity to clear bacterial infection than Ripk $3^{-1-}$ mice, likely through delaying neutrophil constitutive death. Our results clearly suggested that RIPK3/MLKL-mediated necroptosis and GSDMD-mediated pyroptosis contributed to sepsis-induced systemic inflammatory response and multiple organ dysfunction in a cooperative fashion. However, since Ripk3/ Gsdmd or $M l k l / G s d m d$ still could not completely prevent mortality and lethal tissue damage in this CLP sepsis model, other cell death and inflammation signaling may be involved in this complicated process.

Cell death and inflammation occurs in immune cells and nonimmune cells. We further investigated the role for collaboration of RIPK3 and GSDMD in myeloid and nonmyeloid cells during bacterial sepsis progression. Our results demonstrated that Ripk3/Gsdmd-dko $\rightarrow$ WT chimeras recovered from CLP-induced shock and organ injury to certain extent; WT $\rightarrow$ Ripk3/Gsdmd-dko chimeras showed better protection than Ripk3/Gsdmd-dko $\rightarrow$ WT chimeras. Interestingly, the level of inflammatory cytokines is higher in WT $\rightarrow$ Ripk3/Gsdmd-dko chimeras than in Ripk3/Gsdmd-dko $\rightarrow$ WT chimeras. It appears that (i) both RIPK3 and GSDMD in intrinsic tissue cells and inflammatory cells were involved in bacterial sepsis-induced organ injury; (ii) myeloid cells have more significant impact on cytokines production, while nonmyeloid cells play predominant role in tissue injury.

Since the role of macrophage activation and EC dysfunction has been identified in sepsis, we further investigate how Ripk3 and Gsdmd in myeloid cells and nonmyeloid cells collaborated to cause tissue damage. LPS-induced shock and organ damage require inflammatory cytokine signaling via TNF $\alpha$ and IFN $\beta$, which cooperatively drive different modes of cell death to amply inflammation and execute tissue injury [28, 33, 43-45]. We found that Ripk $3^{-/}$Gs $m d^{-/-}$macrophages could inhibit nigericin and intracellular LPS-induced pyroptosis and suppress cleavage of IL-1 $\beta$ for release as well as attenuate TNF $\alpha$-induced cell 
a

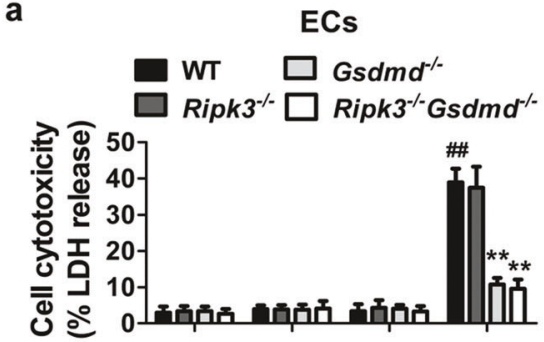<smiles>C=CC=C</smiles>

d

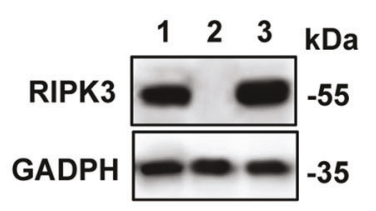

1. $W T+$ Vector

2. Ripk $3^{-}+$Vector

3. Ripk3 $\%+$ Ripk3-Flag

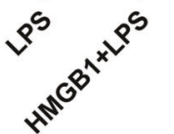

e

b

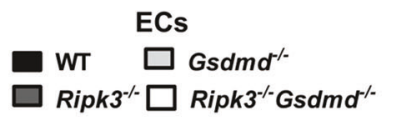
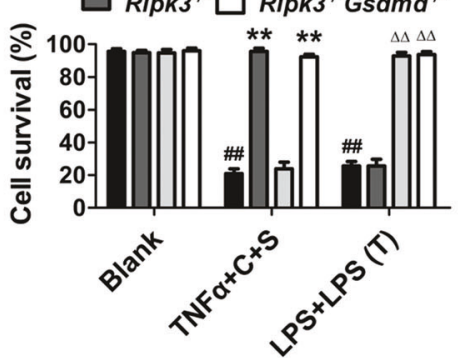

c

ECs
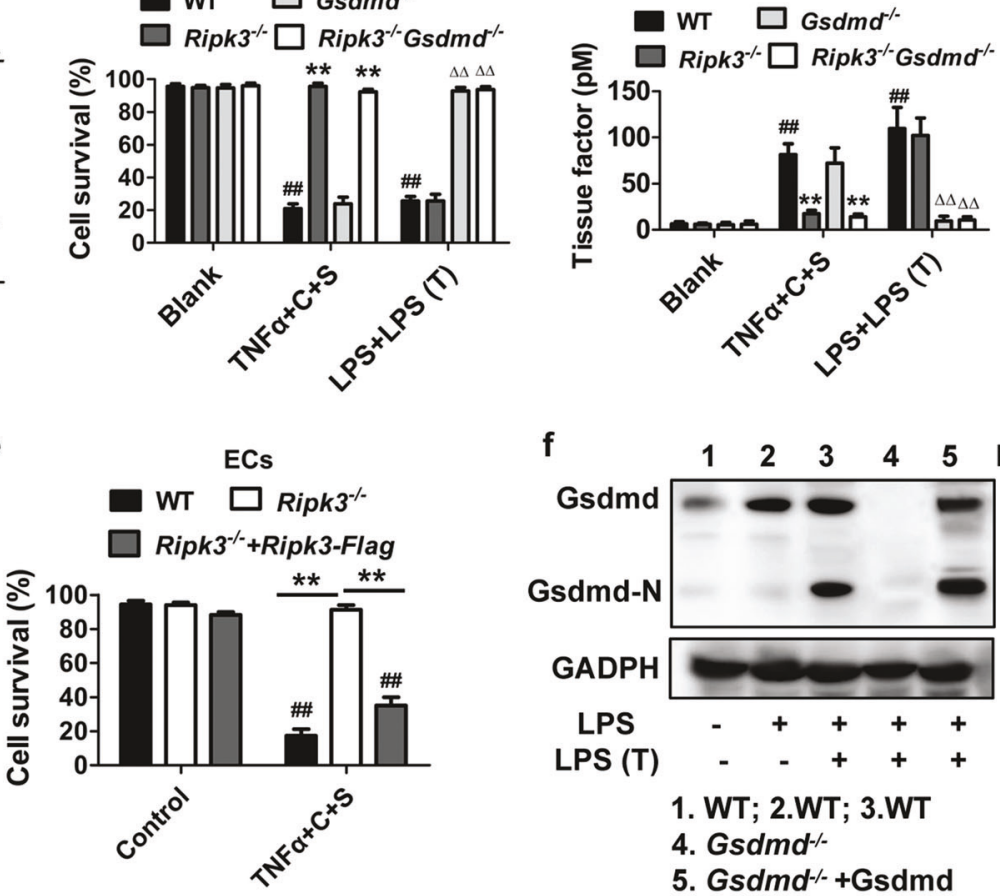

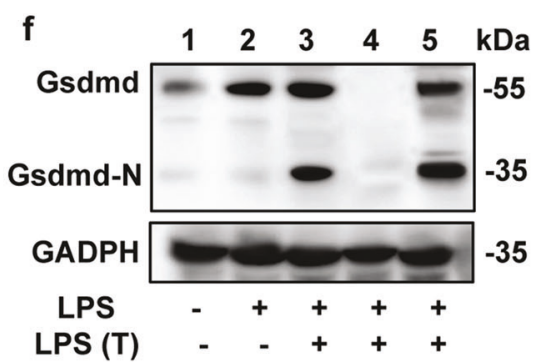

1. WT; 2.WT; 3.WT

5. $G s d m d^{-/-}+G s d m d$
4. $G s d m d^{-/}$ g

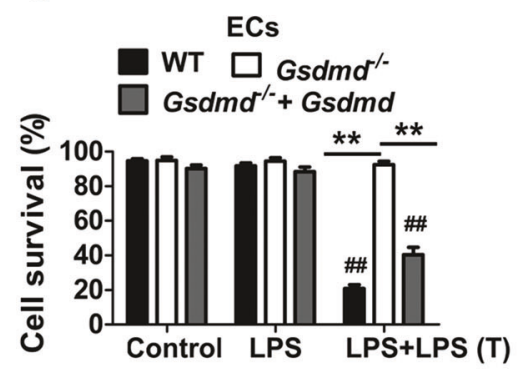

h

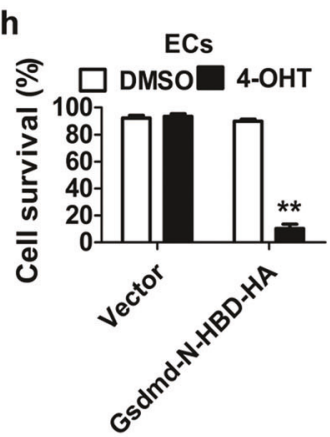

i

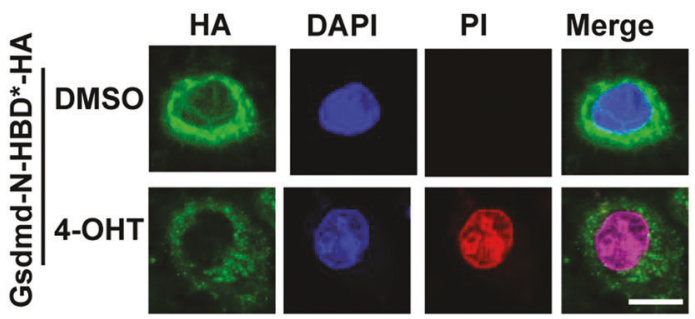

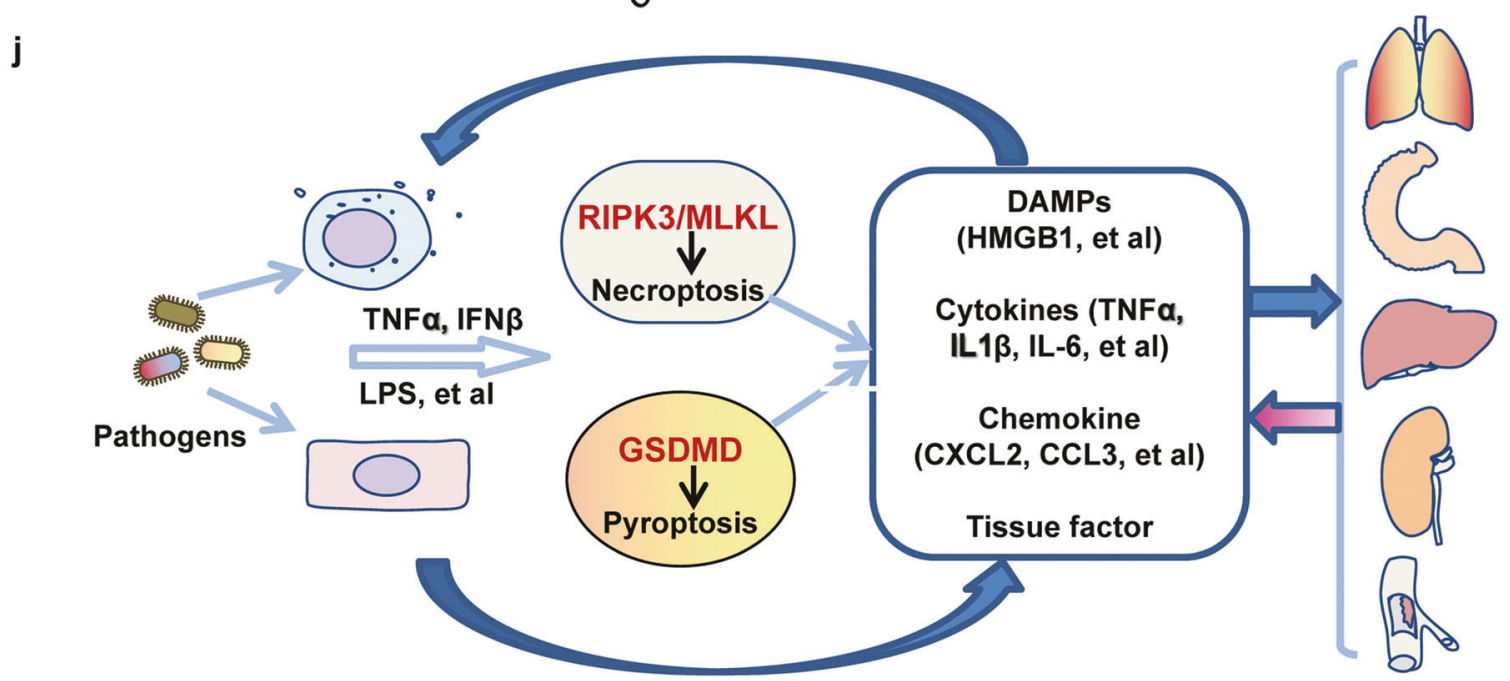

death, suggesting that macrophages lacking Ripk3 and Gsdmd resist to both necroptotic and pyroptotic stimuli and showed stronger defect in proinflammatory cytokines production and release. Interestingly, similar results were obtained in Mlkl-deficient macrophages. Thus, it is reasonable to conceive that both TNF $\alpha$-provoked necroptosis 
Fig. 8 Double deletion of RIPK3 and GSDMD in ECs resisted both necroptosis and pyroptosis. Endothelial cells (ECs) were obtained from the lung of WT, Ripk $3^{-/-}$, Gsdmd ${ }^{-/-}$, and Ripk ${ }^{-/-}$Gsdmd ${ }^{-/-}$ mice. $n=4$. a ECs were incubated with $2 \mu \mathrm{g} / \mathrm{ml} \mathrm{LPS} \mathrm{and} 500 \mathrm{ng} / \mathrm{ml}$ HMGB1 for $20 \mathrm{~h}$. LDH level was analyze for cell cytotoxicity. ${ }^{\#} P<$ 0.01 vs control WT or HMGB1 WT or LPS WT group, $* * P<0.01$ vs HMGB1 + LPS WT group. b, c ECs were treated with $30 \mathrm{ng} / \mathrm{ml}$ mTNF $\alpha$ plus $10 \mu \mathrm{g} / \mathrm{ml} \mathrm{CHX}$ and $10 \mu \mathrm{M}$ SMAC-mimetic $(\mathrm{TNF} \alpha+\mathrm{T}$ $+\mathrm{S}$ ) or $24 \mathrm{~h}$, or transfected with $2 \mu \mathrm{g} / \mathrm{ml}$ LPS for $18 \mathrm{~h}$ after $500 \mathrm{ng} / \mathrm{ml}$ LPS primed for $3 \mathrm{~h}$. Cell survival (b) and TF (c) was measured as described Fig. 7e, f, respectively. ${ }^{\# \#} P<0.01$ vs blank (Non treatment) WT group, ${ }^{* *} P<0.01$ vs $\mathrm{TNF} \alpha+\mathrm{C}+\mathrm{S}$ WT group, ${ }^{\Delta \Delta} P<0.01$ vs LPS + LPS (T) WT group. d, e ECs were infected with lentivirus encoding vector or RIPK3-Flag, and then treated with TNF $\alpha$ plus CHX and Smac (TNF $\alpha+\mathrm{T}+\mathrm{S})$. The levels of RIPK3 (d) was detected by Western blot. Cell survival (e) was measured as described Fig. 7f. ${ }^{\#} P<0.01$ vs Control WT group, $* * P<0.01$. f, g ECs were infected with lentivirus encoding vector or GSDMD. Cells were treated with $500 \mathrm{ng} / \mathrm{ml}$ LPS for $3 \mathrm{~h}$ and then transfected with PBS or 2 $\mu \mathrm{g} / \mathrm{ml}$ LPS for $18 \mathrm{~h}$. GSDMD (f) was detected by Western blot. Cell survival (g) was measured as described Fig. 7a. ${ }^{\# \#} P<0.01$ vs LPS WT group, $* * P<0.01$. h, i ECs were infected with lentivirus encoding GSDMD-N-HBD-HA and then stimulated with DMSO or $1 \mu \mathrm{M} 4-$ OHT for $1 \mathrm{~h}$. Cell survival was assessed in $\mathbf{h}$. Cells were immunostained for HA and counterstained with Hoechst and PI. Representative images were shown as i. (Scale bar $=20 \mu \mathrm{m}$ ). $* * P<0.01$ vs $4-\mathrm{OHT}$ Vector group. All the data are representative of three independent experiments. j Schematic model for the lethal sepsis-induced multiple organ injury via the collaboration between RIPK3/MLKL-dependent necroptosis and GSDMD-dependent pyroptosis.

and LPS-triggered pyroptosis in inflammatory cells collaborated to drive and enhance inflammation activation during sepsis progression. It has been well established that vascular endothelium barrier disruption leads to increased vascular permeability and overproduction of proinflammatory cytokines, which further promote inflammatory cell migration and infiltration into organs to initiate and/or exacerbate tissue injury $[18,20,46]$. In this study, we demonstrated that both pyroptosis triggered by intracellular LPS and necroptosis induced by TNF $\alpha$ could increase cell permeability in ECs. Importantly, double deletion of Ripk3/ Gsdmd or $M l k l / G s d m d$ abolished cell death and permeability induced by either LPS or TNF $\alpha$ in ECs. In addition, inflammatory cell infiltration to tissue was further attenuated by double ablation of Ripk3 and Gsdmd as compared with their single ablation, indicating that RIPK3 and GSDMD contributed together to the induction and progression of endothelium barrier disruption by promoting EC necroptosis and pyroptosis, which facilitated inflammatory cell mobilization into the tissue during bacterial sepsis. Notably, RIPK3/MLKL signaling and GSDMD contributed to activation of the clotting cascade under different stimuli via promoting TF activity from macrophages and ECs, which plays an important role in the progression of tissue injury.

Interestingly, here we demonstrated that HMGB1, increased in bacterial sepsis, was significantly by Gsdmd or Ripk3 or Mlkl deletion. It has been recently shown that
HMGB1 facilitates the entry of LPS into cytoplasm and activate caspase 11-mediated noncanonical pyroptosis $[21,38]$. This implicates that DAMPs, such as HMGB1, released from the necroptotic or pyroptotic cells could enhance macrophage and EC activation and promote inflammation in an autoamplification loop. In addition, cytokines produced by macrophages increased EC permeability, which was inhibited by Ripk3 and Gsdmd double deficiency. These data further support the idea that necroptosis and pyroptosis exert cooperative function to the pathogenesis of sepsis. Thus, our data greatly supported the concept, as modeled in Fig. 8j, that the crosstalk among the inflammatory cells and tissue-residing cells (especially macrophages and ECs) undergoing necroptosis and pyroptosis contributes to bacterial sepsis-induced multi-organ injury.

In summary, we have demonstrated that both RIPK3MLKL-mediated necroptosis and GSDMD-mediated are required to execute sepsis-induced systemic inflammatory response and multi-organ dysfunction, and that combined ablation of Ripk3 and Gsdmd provides better protection against sepsis-triggered tissue injury and mortality. The results from this study may provide new insights into the molecular mechanism of septic pathophysiology and a new indication for treatment of this highly lethal disease.

Acknowledgements This work was supported by grants from National Natural Science Foundation of China (No. 81600525 and No. 81870472), Fujian Provincial Health and Family Planning Commission (No. 2018-CX-24), Joint Funds for the Innovation of Science and Technology of Fujian province (No. 2016Y9014) and Startup Fund for Scientific Research of Fujian Medical University (No. 2017XQ2042). We are grateful to Prof. Jiahuai Han for research information and experimental materials.

\section{Compliance with ethical standards}

Conflict of interest The authors declare that they have no conflict of interest.

Publisher's note Springer Nature remains neutral with regard to jurisdictional claims in published maps and institutional affiliations.

\section{References}

1. Fleischmann C, Scherag A, Adhikari NK, Hartog CS, Tsaganos T, Schlattmann $\mathrm{P}$, et al. Assessment of global incidence and mortality of hospital-treated sepsis. Current estimates and limitations. Am J Respir Crit Care Med. 2016;193:259-72.

2. Joyce DE, Nelson DR, Grinnell BW. Leukocyte and endothelial cell interactions in sepsis: relevance of the protein $\mathrm{C}$ pathway. Crit Care Med. 2004;32:S280-6.

3. Kaukonen KM, Bailey M, Pilcher D, Cooper DJ, Bellomo R. Systemic inflammatory response syndrome criteria in defining severe sepsis. N Engl J Med. 2015;372:1629-38.

4. Rhodes A, Evans LE, Alhazzani W, Levy MM, Antonelli M, Ferrer R, et al. Surviving sepsis campaign: International Guidelines for Management of Sepsis and Septic Shock: 2016. Intensive Care Med. 2017;43:304-77. 
5. Balk RA. Systemic inflammatory response syndrome (SIRS): where did it come from and is it still relevant today? Virulence. 2014;5:20-6.

6. Arcaroli J, Fessler MB, Abraham E. Genetic polymorphisms and sepsis. Shock. 2005;24:300-12.

7. Simmons J, Pittet JF. The coagulopathy of acute sepsis. Curr Opin Anaesthesiol. 2015;28:227-36.

8. Delano MJ, Ward PA. The immune system's role in sepsis progression, resolution, and long-term outcome. Immunol Rev. 2016;274:330-53.

9. Minasyan H. Sepsis: mechanisms of bacterial injury to the patient. Scand J Trauma Resusc Emerg Med. 2019;27:19.

10. Frank D, Vince JE. Pyroptosis versus necroptosis: similarities, differences, and crosstalk. Cell Death Differ. 2019;26:99-114.

11. Weinlich R, Oberst A, Beere HM, Green DR. Necroptosis in development, inflammation and disease. Nat Rev Mol Cell Biol. 2017;18:127-36.

12. Silke J, Rickard JA, Gerlic M. The diverse role of RIP kinases in necroptosis and inflammation. Nat Immunol. 2015;16:689-97.

13. Liu X, Lieberman J. A mechanistic understanding of pyroptosis: the fiery death triggered by invasive infection. Adv Immunol. 2017;135:81-117.

14. Menu P, Vince JE. The NLRP3 inflammasome in health and disease: the good, the bad and the ugly. Clin Exp Immunol. 2011;166:1-15.

15. Brault M, Oberst A. Controlled detonation: evolution of necroptosis in pathogen defense. Immunol Cell Biol. 2017;95:131-6.

16. Han J, Zhong CQ, Zhang DW. Programmed necrosis: backup to and competitor with apoptosis in the immune system. Nat Immunol. 2011;12:1143-9.

17. Wu XN, Yang ZH, Wang XK, Zhang Y, Wan H, Song Y, et al. Distinct roles of RIP1-RIP3 hetero- and RIP3-RIP3 homo-interaction in mediating necroptosis. Cell Death Differ. 2014;21:1709-20.

18. Duprez L, Takahashi N, Van Hauwermeiren F, Vandendriessche B, Goossens V, Vanden Berghe T, et al. RIP kinase-dependent necrosis drives lethal systemic inflammatory response syndrome. Immunity. 2011;35:908-18.

19. Linkermann A, Brasen JH, De Zen F, Weinlich R, Schwendener RA, Green DR, et al. Dichotomy between RIP1- and RIP3mediated necroptosis in tumor necrosis factor-alpha-induced shock. Mol Med. 2012;18:577-86.

20. Zelic M, Roderick JE, O'Donnell JA, Lehman J, Lim SE, Janardhan HP, et al. RIP kinase 1-dependent endothelial necroptosis underlies systemic inflammatory response syndrome. J Clin Invest. 2018;128:2064-75.

21. Kayagaki N, Stowe IB, Lee BL, O'Rourke K, Anderson K, Warming $\mathrm{S}$, et al. Caspase-11 cleaves gasdermin D for noncanonical inflammasome signalling. Nature. 2015;526:666-71.

22. Rittirsch D, Huber-Lang MS, Flierl MA, Ward PA. Immunodesign of experimental sepsis by cecal ligation and puncture. Nat Protoc. 2009;4:31-6.

23. Chen H, Fang Y, Wu J, Chen H, Zou Z, Zhang X, et al. RIPK3MLKL-mediated necroinflammation contributes to AKI progression to CKD. Cell Death Dis. 2018;9:878.

24. Xu Y, Ma H, Shao J, Wu J, Zhou L, Zhang Z, et al. A role for tubular necroptosis in cisplatin-induced AKI. J Am Soc Nephrol. 2015;26:2647-58.

25. Zhang DW, Shao J, Lin J, Zhang N, Lu BJ, Lin SC, et al. RIP3, an energy metabolism regulator that switches TNF-induced cell death from apoptosis to necrosis. Science. 2009;325:332-6.

26. He WT, Wan H, Hu L, Chen P, Wang X, Huang Z, et al. Gasdermin $\mathrm{D}$ is an executor of pyroptosis and required for interleukin1beta secretion. Cell Res. 2015;25:1285-98.
27. Conos SA, Chen KW, De Nardo D, Hara H, Whitehead L, Nunez $\mathrm{G}$, et al. Active MLKL triggers the NLRP3 inflammasome in a cell-intrinsic manner. Proc Natl Acad Sci USA. 2017;114:E961-9.

28. Wallach D, Kang TB, Dillon CP, Green DR. Programmed necrosis in inflammation: toward identification of the effector molecules. Science. 2016;352:aaf2154.

29. Wang YC, Liu QX, Zheng Q, Liu T, Xu XE, Liu XH, et al. Dihydromyricetin alleviates sepsis-induced acute lung injury through inhibiting NLRP3 inflammasome-dependent pyroptosis in mice model. Inflammation. 2019;42:1301-10.

30. Shashaty MGS, Reilly JP, Faust HE, Forker CM, Ittner CAG, Zhang PX, et al. Plasma receptor interacting protein kinase-3 levels are associated with acute respiratory distress syndrome in sepsis and trauma: a cohort study. Crit Care. 2019;23:235.

31. Albelda SM, Smith CW, Ward PA. Adhesion molecules and inflammatory injury. Faseb J. 1994;8:504-12.

32. Clark PR, Manes TD, Pober JS, Kluger MS. Increased ICAM-1 expression causes endothelial cell leakiness, cytoskeletal reorganization and junctional alterations. $J$ Invest Dermatol. 2007; 127:762-74.

33. Mandal P, Feng Y, Lyons JD, Berger SB, Otani S, DeLaney A, et al. Caspase- 8 collaborates with caspase- 11 to drive tissue damage and execution of endotoxic shock. Immunity. 2018;49:42-55.e6.

34. Gando S, Levi M, Toh CH. Disseminated intravascular coagulation. Nat Rev Dis Prim. 2016;2:16037.

35. Vince JE, Wong WW, Gentle I, Lawlor KE, Allam R, O'Reilly L, et al. Inhibitor of apoptosis proteins limit RIP3 kinase-dependent interleukin-1 activation. Immunity. 2012;36:215-27.

36. Zhang Z, Meszaros G, He WT, Xu Y, De Fatima Magliarelli H, Mailly L, et al. Protein kinase D at the Golgi controls NLRP3 inflammasome activation. J Exp Med. 2017;214:2671-93.

37. Wu C, Lu W, Zhang Y, Zhang G, Shi X, Hisada Y, et al. Inflammasome activation triggers blood clotting and host death through pyroptosis. Immunity. 2019;50:1401-11.e4.

38. Deng M, Tang Y, Li W, Wang X, Zhang R, Zhang X, et al. The endotoxin delivery protein HMGB1 mediates caspase-11dependent lethality in sepsis. Immunity. 2018;49:740-53.e7.

39. Cheng KT, Xiong S, Ye Z, Hong Z, Di A, Tsang KM, et al. Caspase-11-mediated endothelial pyroptosis underlies endotoxemiainduced lung injury. J Clin Invest. 2017;127:4124-35.

40. Chen X, He WT, Hu L, Li J, Fang Y, Wang X, et al. Pyroptosis is driven by non-selective gasdermin-D pore and its morphology is different from MLKL channel-mediated necroptosis. Cell Res. 2016;26:1007-20.

41. Dickson K, Lehmann C. Inflammatory response to different toxins in experimental sepsis models. Int J Mol Sci. 2019;20: 4341.

42. Zanoni I, Tan Y, Di Gioia M, Broggi A, Ruan J, Shi J, et al. An endogenous caspase-11 ligand elicits interleukin-1 release from living dendritic cells. Science. 2016;352:1232-6.

43. Karaghiosoff M, Steinborn R, Kovarik P, Kriegshauser G, Baccarini M, Donabauer B, et al. Central role for type I interferons and Tyk2 in lipopolysaccharide-induced endotoxin shock. Nat Immunol. 2003;4:471-7.

44. Lawlor KE, Khan N, Mildenhall A, Gerlic M, Croker BA, D’Cruz AA, et al. RIPK3 promotes cell death and NLRP3 inflammasome activation in the absence of MLKL. Nat Commun. 2015;6:6282.

45. Tonnus W, Gembardt F, Latk M, Parmentier S, Hugo C, Bornstein $\mathrm{SR}$, et al. The clinical relevance of necroinflammationhighlighting the importance of acute kidney injury and the adrenal glands. Cell Death Differ. 2019;26:68-82.

46. Nourshargh S, Alon R. Leukocyte migration into inflamed tissues. Immunity. 2014;41:694-707. 